\title{
Multileptonic signals of co-annihilating left-right supersymmetric dark matter
}

\author{
Arindam Chatterjee* \\ Indian Statistical Institute, 203 B.T. Road, Kolkata-700108, India \\ Mariana Frank ${ }^{\dagger}$ \\ Department of Physics, Concordia University, \\ 7141 Sherbrooke St.West, Montreal H4B 1R6, QC, Canada \\ Benjamin Fuks \\ Sorbonne Université, CNRS, Laboratoire de Physique Théorique et Hautes Énergies, \\ LPTHE, F-75005 Paris, France, \\ and Institut Universitaire de France, 103 boulevard Saint-Michel, 75005 Paris, France \\ Katri Huitu ${ }^{\S}$ and Subhadeep Mondal \\ Department of Physics, and Helsinki Institute of Physics, \\ P. O. Box 64, FI-00014 University of Helsinki, Finland \\ Santosh Kumar Rai" \\ Regional Centre for Accelerator-based Particle Physics, Harish-Chandra Research Institute, \\ HBNI, Chhatnag Road, Jhusi, Allahabad 211019, India \\ Harri Waltari ${ }^{* *}$ \\ Department of Physics and Astronomy, University of Southampton, \\ Highfield, Southampton SO17 1BJ, United Kingdom, \\ and Department of Physics, and Helsinki Institute of Physics, \\ P. O. Box 64, FI-00014 University of Helsinki, Finland
}

(Received 22 October 2018; published 15 February 2019)

\begin{abstract}
We perform a comprehensive dark matter analysis of left-right supersymmetric scenarios that includes constraints from dark matter direct and indirect detection experiments and that presents distinctive features from those available in minimal supersymmetry. We concentrate on dark matter candidates which, while satisfying all constraints, are different from those of the minimal supersymmetric standard model. We consider in our analysis all possible co-annihilation channels relevant for setups in which several states are light and nearly degenerate, and devise a set of representative benchmark points, requiring co-annihilations, which satisfy all restrictions. We then study their consequent LHC signals, which exhibit promising new multileptonic signatures involving $W_{R}$, that if observed, would provide a strong support for left-right supersymmetry.
\end{abstract}

DOI: 10.1103/PhysRevD.99.035017

*arindam.chatterjee@gmail.com

†mariana.frank@concordia.ca

\#uks@1pthe.jussieu.fr

\$katri.huitu@helsinki.fi

"subhadeep.mondal@helsinki.fi

skrai@hri.res.in

*harri.waltari@helsinki.fi

Published by the American Physical Society under the terms of the Creative Commons Attribution 4.0 International license. Further distribution of this work must maintain attribution to the author(s) and the published article's title, journal citation, and DOI. Funded by SCOAP ${ }^{3}$.

\section{INTRODUCTION}

The LHC experiments have probed the standard model (SM) at high energies with no clear signs of new physics so far. Nevertheless, it is well known that the SM needs to be extended, as neutrino oscillation experiments show that neutrinos have masses [1-5] and there is convincing evidence for cold dark matter from galaxy rotation curves $[6,7]$, the cosmic microwave background [8] and the Bullet cluster observations [9]. In addition, the SM has features that do not have a proper explanation, like for instance parity violation and the strong $C P$ problem. 
In the SM, the Higgs boson mass term gets quadratic corrections that are proportional to the scale of new physics, so that we expect some kind of a cutoff mechanism to exist not too far above the electroweak scale. Supersymmetry (SUSY) offers such a mechanism as the quadratic corrections stemming from bosonic and fermionic states cancel, even if SUSY is softly broken.

Within the framework of SUSY, left-right symmetric (LR) models $[10,11]$ have attractive features. As they are based on the $S U(3)_{\mathrm{C}} \times S U(2)_{\mathrm{L}} \times S U(2)_{\mathrm{R}} \times U(1)_{B-L}$ gauge group, $R$-parity violation is forbidden as it would break $U(1)_{B-L}$. In addition, parity breaking is dynamical and the strong $C P$ problem gets solved as the parity violating QCD $\theta$-term is absent at tree-level and is only generated at the two-loop level [12-14]. Moreover, LR symmetry requires the existence of right-handed neutrinos, so that neutrinos are naturally massive, although the actual implementation of a seesaw mechanism is not straightforward as right-handed neutrino bare mass terms are forbidden by the model gauge symmetry. The usual solution requires the presence of chiral $S U(2)_{\mathrm{R}}$ triplet superfields with nonzero $B-L$ quantum numbers. Their neutral scalar components then break lepton number spontaneously, which generates right-handed neutrino mass terms.

Breaking parity with $S U(2)_{\mathrm{R}}$ triplets leads to a tree-level scalar potential that violates either charge conservation or $R$-parity invariance [15]. The former is unacceptable and the latter makes the lightest supersymmetric particle (LSP) unstable, so that it could not be a viable dark matter (DM) candidate anymore. Without extending the particle content further, the charge and $R$-parity conserving minimum can, however, be stabilized by including one-loop corrections to the scalar potential $[16,17]$. This both saves the LSP as a viable DM candidate and also forces the LR symmetry breaking scale to be relatively low, the latter yielding hopes of finding left-right supersymmetry (LRSUSY) at the LHC.

LRSUSY has a number of viable DM candidates. The model has twelve neutralinos, and both gaugino-dominated and Higgsino-dominated states are acceptable DM candidates. In addition, right-handed sneutrinos may annihilate efficiently enough through gauge interactions to satisfy the relic density constraints from Planck without the need of mixing left- and right-handed sneutrino states. In a previous work [18], we have analyzed right-handed sneutrinos and gauginos LSP as candidates for dark matter, but when the LSP is much lighter than the next-to-lightest superpartner (NLSP) so that coannihilations can be ignored. In this work, we relax this assumption and extend our analysis to other possibilities where co-annihilation channels matter. As the model features two Higgs bidoublets, there are generally several nearly-degenerate Higgsino-like neutralinos and charginos with a mass close to the effective off-diagonal Higgs mass mixing parameter $\mu_{\text {eff }}$ Co-annihilations are hence always present and relevant both for a Higgsinolike DM candidate and when the LSP is close in mass of the Higgsinos. We furthermore also examine prospects for DM indirect detection, especially in the view of a right-handed sneutrino LSP annihilating into right-handed neutrinos.

We finally study how these scenarios could emerge through multilepton production in association with missing energy at the LHC, a collider signature that could give strong support for the realization of LRSUSY in nature. In practice, we use DM relic density constraints to fix the masses of the LSP and of the co-annihilating neutralinos and charginos, and investigate, for a few representative benchmark scenarios, the production and decay of a not too heavy $S U(2)_{\mathrm{R}} W_{R}$ boson into charginos and neutralinos. Such a channel is usually linked to a sizeable branching fraction into leptons and missing energy, so that the corresponding new physics signal can be constrained by typical electroweakino searches. We therefore analyze the sensitivity of a recent CMS SUSY search in the multileptons plus missing energy channel [19] and then estimate the prospects of the high-luminosity phase of the LHC.

Our work is organized as follows. We give a brief introduction to the LRSUSY model version considered in Sec. II. In Sec. III we proceed to consider existing constraints from collider experiments and dark matter searches. We then select a number of benchmark points representing different dark matter motivated model configurations in Sec. IV and then analyze the prospects of DM indirect detection in Sec. V, and of collider searches in Sec. VI. We summarize our findings and conclude in Sec. VII.

\section{THE LEFT-RIGHT SUPERSYMMETRIC MODEL}

Left-right supersymmetric models, based on the $S U(3)_{\mathrm{C}} \times S U(2)_{\mathrm{L}} \times S U(2)_{\mathrm{R}} \times U(1)_{B-L}$ gauge symmetry, inherit all attractive features of the left-right symmetry $[10,11]$, whereas they forbid, thanks to the gauged $B-L$ symmetry, any $R$-parity violating operators problematic in the minimal supersymmetric standard model (MSSM). The chiral matter in LRSUSY consist of three families of quark and lepton superfields,

$$
\begin{aligned}
Q_{L} & =\left(\begin{array}{c}
u_{L} \\
d_{L}
\end{array}\right) \sim\left(\mathbf{3}, \mathbf{2}, \mathbf{1}, \frac{1}{3}\right), \\
Q_{R} & =\left(\begin{array}{c}
d_{R} \\
-u_{R}
\end{array}\right) \sim\left(\overline{\mathbf{3}}, \mathbf{1}, \mathbf{2}^{*},-\frac{1}{3}\right), \\
L_{L} & =\left(\begin{array}{c}
\nu_{L} \\
e_{L}
\end{array}\right) \sim(\mathbf{1}, \mathbf{2}, \mathbf{1},-1), \\
L_{R} & =\left(\begin{array}{c}
e_{R} \\
-N
\end{array}\right) \sim\left(\mathbf{1}, \mathbf{1}, \mathbf{2}^{*}, 1\right),
\end{aligned}
$$


where the numbers in the brackets denote the representation under the $S U(3)_{\mathrm{C}}, S U(2)_{\mathrm{L}}, S U(2)_{\mathrm{R}}$ and $U(1)_{B-L}$ gauge factors. To ascribe a small magnitude of the neutrino masses and preserve $R$-parity, the model superfield content includes both $S U(2)_{\mathrm{L}}$ and $S U(2)_{\mathrm{R}}$ triplets of Higgs supermultiplets, in addition to two Higgs bidoublets and one singlet,

$$
\begin{aligned}
\Phi_{1} & =\left(\begin{array}{ll}
\Phi_{11}^{+} & \Phi_{11}^{0} \\
\Phi_{12}^{0} & \Phi_{12}^{-}
\end{array}\right) \sim\left(\mathbf{1}, \mathbf{2}, \mathbf{2}^{*}, 0\right), \\
\Phi_{2} & =\left(\begin{array}{ll}
\Phi_{21}^{+} & \Phi_{21}^{0} \\
\Phi_{22}^{0} & \Phi_{22}^{-}
\end{array}\right) \sim\left(\mathbf{1}, \mathbf{2}, \mathbf{2}^{*}, 0\right), \\
\Delta_{L} & =\left(\begin{array}{cc}
\frac{1}{\sqrt{2}} \Delta_{L}^{-} & \Delta_{L}^{0} \\
\Delta_{L}^{--} & -\frac{1}{\sqrt{2}} \Delta_{L}^{-}
\end{array}\right) \sim(\mathbf{1}, \mathbf{3}, \mathbf{1},-2), \\
\delta_{L} & =\left(\begin{array}{cc}
\frac{1}{\sqrt{2}} \delta_{L}^{+} & \delta_{L}^{++} \\
\delta_{L}^{0} & -\frac{1}{\sqrt{2}} \delta_{L}^{+}
\end{array}\right) \sim(\mathbf{1}, \mathbf{3}, \mathbf{1}, 2), \\
\Delta_{R} & =\left(\begin{array}{cc}
\frac{1}{\sqrt{2}} \Delta_{R}^{-} & \Delta_{R}^{0} \\
\Delta_{R}^{--} & -\frac{1}{\sqrt{2}} \Delta_{R}^{-}
\end{array}\right) \sim(\mathbf{1}, \mathbf{1}, \mathbf{3},-2), \\
\delta_{R} & =\left(\begin{array}{cc}
\frac{1}{\sqrt{2}} \delta_{R}^{+} & \delta_{R}^{++} \\
\delta_{R}^{0} & -\frac{1}{\sqrt{2}} \delta_{R}^{+}
\end{array}\right) \sim(\mathbf{1}, \mathbf{1}, \mathbf{3}, 2), \\
S & \sim(\mathbf{1}, \mathbf{1}, \mathbf{1}, 0),
\end{aligned}
$$

where the numbers in the brackets again denote the representation under the model gauge group. The superpotential of the model is given by

$$
\begin{aligned}
W= & Q_{L}^{T} \mathbf{Y}_{Q}^{(i)} \Phi_{i} Q_{R}+L_{L}^{T} \mathbf{Y}_{L}^{(i)} \Phi_{i} L_{R}+L_{L}^{T} \mathbf{h}_{L L} \delta_{L} L_{L} \\
& +L_{R}^{T} \mathbf{h}_{R R} \Delta_{R} L_{R}+\lambda_{L} S \operatorname{Sr}\left[\Delta_{L} \delta_{L}\right] \\
& +\lambda_{R} S \operatorname{Tr}\left[\Delta_{R} \delta_{R}\right]+\lambda_{3} S \operatorname{Tr}\left[\tau_{2} \Phi_{1}^{T} \tau_{2} \Phi_{2}\right]+\lambda_{4} S \operatorname{Tr}\left[\tau_{2} \Phi_{1}^{T} \tau_{2} \Phi_{1}\right] \\
& +\lambda_{5} S \operatorname{Tr}\left[\tau_{2} \Phi_{2}^{T} \tau_{2} \Phi_{2}\right]+\lambda_{S} S^{3}+\xi_{F} S,
\end{aligned}
$$

where the Yukawa couplings $\mathbf{Y}_{Q, L}$ and $\mathbf{h}_{L L, R R}$ are $3 \times 3$ matrices in flavor space, the $\lambda$ parameters stand for the strengths of the various Higgs(ino) interactions, with $\tau_{2}$ being the second Pauli matrix, and $\xi$ consists in a linear singlet term. The Lagrangian of the model includes, on top of usual SUSY gauge interaction, kinetic and superpotential interaction terms, soft SUSY-breaking terms (standard scalar and gaugino mass terms together with multiscalar interactions deduced from the form of the superpotential). Explicit expressions and more details about the model can be found in Refs. $[18,20]$.
The neutral component of the $S U(2)_{\mathrm{R}}$ Higgs scalar field $\Delta_{R}$ acquires a large vacuum expectation value (VEV) $v_{R}$, which breaks the LR symmetry and makes the $S U(2)_{\mathrm{R}}$ gauge sector heavy. The complete set of nonvanishing VEVs responsible for breaking the symmetry down to $U(1)_{\mathrm{em}}$ reads

$$
\begin{gathered}
\left\langle\Phi_{1}\right\rangle=\left(\begin{array}{cc}
0 & 0 \\
\frac{v_{1}}{\sqrt{2}} & 0
\end{array}\right), \quad\left\langle\Phi_{2}\right\rangle=\left(\begin{array}{cc}
0 & \frac{v_{2}}{\sqrt{2}} \\
0 & 0
\end{array}\right), \\
\left\langle\Delta_{R}\right\rangle=\left(\begin{array}{cc}
0 & \frac{v_{R}}{\sqrt{2}} \\
0 & 0
\end{array}\right), \quad\left\langle\delta_{R}\right\rangle=\left(\begin{array}{cc}
0 & 0 \\
\frac{v_{R}^{\prime}}{\sqrt{2}} & 0
\end{array}\right), \\
\langle S\rangle=\frac{v_{S}}{\sqrt{2}} .
\end{gathered}
$$

This vacuum structure allows for avoiding constraints from electroweak precision tests, flavor-changing neutral currents and a too large mixing between the $W_{L}$ and $W_{R}$ bosons. It is moreover stable provided that $\lambda_{4}=\lambda_{5}=0$. In order to prevent the tree-level vacuum state from being a charge-breaking one, one can either rely on spontaneous $R$-parity violation [15], one-loop corrections $[16,17]$, higher-dimensional operators [12] or additional $B-L=0$ triplets [21]. Whereas the first two options restrict $v_{R}$ to be of at most about $10 \mathrm{TeV}$, the latter ones enforce $v_{R}$ to lie above $10^{10} \mathrm{GeV}$. In this work, we rely on radiative corrections to stabilize the vacuum, so that the LSP is stable and can act as a DM candidate [18]. Two viable LSP options emerge from LRSUSY, neutralinos and right sneutrinos.

\section{A. Neutralinos and charginos}

The model has twelve neutralinos whose mass matrix can be decomposed into three independent blocks, two $2 \times 2$ blocks describing the mixing of the $\tilde{\delta}_{L} / \tilde{\Delta}_{L}$ and $\tilde{\Phi}_{22}^{0} / \tilde{\Phi}_{11}^{0}$ fields respectively, and one $8 \times 8$ block related to the mixing of the eight other neutral Higgsinos and gauginos. The two dimension-two blocks are given, in the $\left(\tilde{\delta}_{L}^{0}, \tilde{\Delta}_{L}^{0}\right)$ and $\left(\tilde{\Phi}_{22}^{0}, \tilde{\Phi}_{11}^{0}\right)$ bases, by

$$
M_{\tilde{\chi}_{\delta}}=\left(\begin{array}{cc}
0 & \mu_{L} \\
\mu_{L} & 0
\end{array}\right) \text { and } M_{\tilde{\chi}_{\Phi}}=\left(\begin{array}{cc}
0 & -\mu_{\mathrm{eff}} \\
-\mu_{\mathrm{eff}} & 0
\end{array}\right),
$$

while the last $8 \times 8$ block reads, in the $\left(\tilde{\Phi}_{12}^{0}, \tilde{\Phi}_{21}^{0}, \tilde{\delta}_{R}^{0}, \tilde{\Delta}_{R}^{0}\right.$, $\left.\tilde{S}, \tilde{B}, \tilde{W}_{L}^{0}, \tilde{W}_{R}^{0}\right)$ basis, 


$$
M_{\tilde{\chi}^{0}}=\left(\begin{array}{cccccccc}
0 & -\mu_{\mathrm{eff}} & 0 & 0 & -\mu_{d} & 0 & \frac{g_{L} v_{u}}{\sqrt{2}} & -\frac{g_{R} v_{u}}{\sqrt{2}} \\
-\mu_{\mathrm{eff}} & 0 & 0 & 0 & -\mu_{u} & 0 & -\frac{g_{L} v_{d}}{\sqrt{2}} & \frac{g_{R} v_{d}}{\sqrt{2}} \\
0 & 0 & 0 & \mu_{R} & \frac{\lambda_{R} v_{R}^{\prime}}{\sqrt{2}} & g^{\prime} v_{R} & 0 & -g_{R} v_{R} \\
0 & 0 & \mu_{R} & 0 & \frac{\lambda_{R} v_{R}}{\sqrt{2}} & -g^{\prime} v_{R}^{\prime} & 0 & -g_{R} v_{R}^{\prime} \\
-\mu_{d} & -\mu_{u} & \frac{\lambda_{R} v_{R}^{\prime}}{\sqrt{2}} & \frac{\lambda_{R} v_{R}}{\sqrt{2}} & \mu_{S} & 0 & 0 & 0 \\
0 & 0 & g^{\prime} v_{R} & -g^{\prime} v_{R}^{\prime} & 0 & M_{1} & 0 & 0 \\
\frac{g_{L} v_{u}}{\sqrt{2}} & -\frac{g_{L} v_{d}}{\sqrt{2}} & 0 & 0 & 0 & 0 & M_{2 L} & 0 \\
-\frac{g_{R} v_{u}}{\sqrt{2}} & \frac{g_{R} v_{d}}{\sqrt{2}} & -g_{R} v_{R} & -g_{R} v_{R}^{\prime} & 0 & 0 & 0 & M_{2 R}
\end{array}\right) .
$$

In the above expressions, we have defined $\mu_{\text {eff }}=\lambda_{3} \frac{v_{S}}{\sqrt{2}}$, $\mu_{S}=\lambda_{S} \frac{v_{S}}{\sqrt{2}}, \mu_{L, R}=\lambda_{L, R} \frac{v_{S}}{\sqrt{2}}$ and $\mu_{u, d}=\lambda_{3} \frac{v_{u, d}}{\sqrt{2}}$.

We assume that the $\lambda_{R}$ coupling is large, as this helps to stabilize the vacuum state [17]. Invoking LR symmetry, $\lambda_{L}$ is taken large too. After accounting for the fact that $v_{R}, v_{R}^{\prime}$ (responsible for LR breaking) and $v_{S}$ (induced by SUSY breaking) are large as well, it turns out that many of the neutralino states are heavy. Gauginos, whose masses originate in contrast from soft terms, can be light (with the exception of the $\tilde{W}_{R}$ wino that has a mass close to the $W_{R}$ boson mass). The LR bidoublet Higgsinos, which are nearly degenerate with masses close to $\left|\mu_{\text {eff }}\right|$, can also be light. As shown by the non-zero elements of the mass matrix, Eq. (2.5), two of the Higgsinos mix with the $\tilde{W}_{L, R}$ winos with a strength depending on $\tan \beta$. Typically then, Higgsino-dominated states acquire a gaugino component that can be up to $10 \%$. Due to the breaking of the $S U(2)_{\mathrm{R}} \times$ $U(1)_{B-L}$ symmetry into $U(1)_{\mathrm{Y}}$, the $\tilde{B}$ and $\tilde{W}_{R}$ gauginos mix. If one of these admixtures is close in mass to the bidoublet Higgsinos, further mixings occur, as abovementioned, and the degeneracy of the Higgsinos states is lifted. In this case, the gaugino-dominated state becomes further split from the rest of the neutralino spectrum.

In the charged sector, the model has six singly-charged charginos whose mass matrix is written, in the $\left(\tilde{\delta}_{L}^{+}, \tilde{\delta}_{R}^{+}, \tilde{\Phi}_{11}^{+}\right.$, $\left.\tilde{\Phi}_{21}^{+}, \tilde{W}_{L}^{+}, \tilde{W}_{R}^{+}\right)$and $\left(\tilde{\Delta}_{L}^{-}, \tilde{\Delta}_{R}^{-}, \tilde{\Phi}_{12}^{-}, \tilde{\Phi}_{22}^{-}, \tilde{W}_{L}^{-}, \tilde{W}_{R}^{-}\right)$bases as

$$
M_{\tilde{\chi}^{ \pm}}=\left(\begin{array}{cccccc}
\lambda_{L} v_{S} / \sqrt{2} & 0 & 0 & 0 & 0 & 0 \\
0 & \lambda_{R} v_{S} / \sqrt{2} & 0 & 0 & 0 & -g_{R} v_{R} \\
0 & 0 & 0 & \mu_{\mathrm{eff}} & g_{L} v_{u} / \sqrt{2} & 0 \\
0 & 0 & \mu_{\mathrm{eff}} & 0 & 0 & -g_{R} v_{d} / \sqrt{2} \\
0 & 0 & 0 & g_{L} v_{d} / \sqrt{2} & M_{2 L} & 0 \\
0 & g_{R} v_{R}^{\prime} & -g_{R} v_{u} / \sqrt{2} & 0 & 0 & M_{2 R}
\end{array}\right) .
$$

only be accommodated with dominantly-bino-like LSP with a mass close to $m_{h} / 2$ [18], whilst in the bidoublet Higgsino case (featuring four neutralinos and two charginos that are nearly-degenerate), co-annihilations play a crucial role and impose Higgsino masses close to $700 \mathrm{GeV}$. Hence the Higgsino LSP case is an example of a heavy and compressed spectrum, which poses a challenge for direct searches for SUSY.

Right sneutrino LSPs annihilate via the exchange of an $s$ channel Higgs boson through gauge interactions stemming from the $D$-terms [18]. Without options for co-annihilating, the LSP sneutrino mass must lie between 250 and $300 \mathrm{GeV}$, heavier masses leading to DM overproduction. However, potential co-annihilations with neutralinos enhance the 
effective annihilation cross section so that the relic density constraints can be satisfied with heavier sneutrinos. The fully degenerate sneutrino/Higgsino scenario imposes an upper limit on the LSP sneutrino mass of $700 \mathrm{GeV}$. Additionally, together with the LSP, right neutrinos can also be part of the dark sector [22].

As direct detection constraints imposed by the XENON1T [23] and PANDA [24] collaborations have put light DM scenarios under severe scrutiny, viable LRSUSY DM setups accounting for the relic density and direct detection constraints simultaneously need to rely on various co-annihilation options. In this work we consider several of such scenarios with different LSP options, and additionally highlight the corresponding implications for searches at the LHC.

A robust signal of left-right symmetry consists in the discovery of an $S U(2)_{\mathrm{R}}$ gauge boson $W_{R}$, possibly together with a right neutrino $N$. From a pure spectral analysis, the SUSY nature of such a signal could originate from the above dark matter considerations that lead to favored LRSUSY scenarios in which several neutralinos and charginos are light. This hence motivates LRSUSY investigations through a new $W_{R}$ boson search channel, where decays into pairs of electroweakinos are considered, the corresponding branching ratio being as large as $25 \%$ in many LRSUSY scenarios. Especially when a sneutrino is the LSP, we expect that the decays of these neutralinos and charginos lead to leptonic final states at colliders, so that the production of multileptonic systems in association with a large amount of missing transverse energy $E_{T}$ is enhanced. Whilst such a multilepton signal with missing energy is a characteristic SUSY collider signal, it also provides an additional search channel for $W_{R}$ bosons. The resonant production mode offers the opportunity to reconstruct the $W_{R}$ boson mass through the study of kinematic thresholds featured by various transverse observables.

Before proceeding to the analysis of promising collider signals, we review in the next section the pertinent features and constraints imposed on the parameters of the model, including those coming from dark matter.

\section{CONSTRAINTS ON THE SPECTRUM}

\section{A. The Higgs sector}

The considered LRSUSY version has a relatively light $S U(2)_{\mathrm{R}}$ doubly-charged Higgs boson with a mass originating from loop corrections $[16,17,25,26]$. Whereas the ATLAS collaboration has excluded doubly-charged Higgs masses ranging up to $650-760 \mathrm{GeV}$ when the doublycharged Higgs boson decays exclusively into same-sign electrons or muons [27], masses of about $300 \mathrm{GeV}$ are still allowed when the branching ratio in these modes is of at most a few percents. In contrast, the CMS collaboration has searched for doubly-charged Higgs bosons in all leptonic channels but interpreted the results only in the $S U(2)_{\mathrm{L}}$ case.
As the associated production of an $S U(2)_{\mathrm{R}}$ doubly-charged Higgs boson with a singly-charged one is suppressed by the $W_{R}$ mass and as any neutral current production mode is weaker by virtue of reduced couplings, the limit of $396 \mathrm{GeV}$ [28] can be reduced to about $300-350 \mathrm{GeV}$, depending on the branching ratio into tau pairs.

Although one could enforce the doubly-charged Higgs boson to be heavy enough to be compliant with all current bounds, we prefer imposing that it decays mainly into tau leptons. We fix the different branching ratios to

$$
\begin{aligned}
\operatorname{BR}\left(H^{ \pm \pm} \rightarrow \tau^{ \pm} \tau^{ \pm}\right) & =92 \%, \\
\operatorname{BR}\left(H^{ \pm \pm} \rightarrow \mu^{ \pm} \mu^{ \pm}\right) & =\operatorname{BR}\left(H^{ \pm \pm} \rightarrow e^{ \pm} e^{ \pm}\right)=4 \%,
\end{aligned}
$$

so that the mass of the $S U(2)_{\mathrm{R}}$ doubly-charged Higgs boson can be safely set to about $350 \mathrm{GeV}$, the exact value being not crucial for our discussion. The important parameter consists instead in the Yukawa texture, which also determines right-handed neutrino masses and contributes to the sneutrino mass matrix.

We impose that the SM-like Higgs boson mass $m_{h}$ is compatible with $[29,30]$

$$
m_{h}=125.1 \pm 0.3 \mathrm{GeV},
$$

the uncertainty being chosen smaller than the corresponding theoretical error. The Higgs mass is an essential input in the relic density computation, due to s-channel Higgs boson exchange contributions, so that we want that input to be reasonably close to the experimentally-measured value. The SM-like Higgs boson mass is mostly affected by $\tan \beta$ and the stop masses and mixings. As the tree-level mass is larger than in the MSSM [31,32], a $125 \mathrm{GeV}$ mass value can always be achieved with rather moderate stop masses and mixings. However, $\tan \beta$ cannot have too small a value as the tree-level Higgs mass vanishes in the $\tan \beta \rightarrow 1$ limit, like in the MSSM. With $\tan \beta \gtrsim 5$, we obtain a SM-like Higgs boson mass compatible with the experimental value, for stop masses of a couple of $\mathrm{TeV}$.

The second $C P$-even state, the lightest $C P$-odd state and the lightest charged Higgs bosons are predicted to be nearly degenerate and of mass of about $m_{A}$. The most stringent constraint on $m_{A}$ comes from the $B_{s} \rightarrow \mu \mu$ decay, to which the $C P$-odd state can yield a sizeable contribution, which is enhanced for large values of $\tan \beta$ [33]. We therefore use moderate values for $\tan \beta$. Starting from

$$
m_{A}^{2} \sim g_{R}^{2} v_{R}^{2}\left(\tan ^{2} \beta_{R}-1\right),
$$

we correlate $v_{R}$ and $\tan \beta_{R}$ so that the resulting masses are close to $650 \mathrm{GeV}$, a value that is in addition compatible with direct search results. In those notations, $\tan \beta_{R}$ stands for the ratio of the two $S U(2)_{\mathrm{R}}$ Higgs triplet VEVs and $g_{R}$ for the $S U(2)_{\mathrm{R}}$ coupling constant. On the other hand, if $\tan \beta_{R}$ deviates too much from 1 , it gives a negative contribution to 
the doubly-charged Higgs-boson mass, thus we impose values close to 1.05 for $\tan \beta_{R}$.

All other Higgs bosons have masses of the scale of either $v_{R}$ or $v_{S}$, i.e., of several $\mathrm{TeV}$, so they do not yield any constraint on the model.

\section{B. Right-handed neutrinos}

Generic searches for right-handed neutrinos were performed at LEP [34], leading to bounds on right-handed Majorana neutrino masses of at most $90.7 \mathrm{GeV}$. In our model the right-handed neutrino mass matrix reads

$$
\left(\mathbf{m}_{\mathbf{N}}\right)_{i j}=\left(\mathbf{h}_{R R}\right)_{i j} v_{R},
$$

where $\mathbf{h}_{R R}$ also dictates the different doubly-charged Higgs branching ratio. As we have enforced the $S U(2)_{\mathrm{R}}$ doublycharged Higgs boson to decay mainly into taus, the righthanded tau neutrino turns out to be significantly heavier than the others. With our choices of $v_{R}$ (essentially determined by the bounds on the $W_{R}$ boson mass as shown in Sec. III C), the electron and muon right-handed neutrino masses are close to $150 \mathrm{GeV}$, whereas the right-handed tau neutrino mass is of about $750 \mathrm{GeV}$.

Such a spectrum implies that $t$-channel neutralinomediated sneutrino DM annihilation into right-handed neutrinos is kinematically open only for electron or muon sneutrinos, but not for the tau ones, once the mass constraints originating from the DM relic density are accounted for (see Sec. III D).

\section{The $S U(2)_{\mathrm{R}}$ gauge sector}

In LRSUSY, the masses of the $W_{R}$ and $Z_{R}$ bosons are related and the $Z_{R}$ is always heavier than the $W_{R}$ boson. Hence $W_{R}$ searches are more restrictive. The $W_{R}$ boson can decay into jets and, if the $S U(2)_{\mathrm{R}}$ gauge coupling equals the $S U(2)_{\mathrm{L}}$ one $\left(g_{L}=g_{R}\right)$, limits on sequential $W^{\prime}$ bosons can be reinterpreted straightforwardly. ATLAS and CMS have obtained bounds of 3.6 TeV [35] and 3.3 TeV [36], on such a sequential extra gauge boson, respectively. After taking into account the $20 \%-25 \%$ chance that the $W_{R}$ boson decays into superpartners, bounds turn to be slightly weaker. We conservatively adopt, in our analysis, a $W_{R}$ mass,

$$
m_{W_{R}} \gtrsim 3.3 \mathrm{TeV},
$$

that allows for evading those constraints. For such values of $W_{R}$-boson masses, the corresponding neutral $Z_{R}$ boson is heavier, with a mass of about $5.6 \mathrm{TeV}$. This is compatible with the current experimental lower bounds for $Z^{\prime}$ bosons decaying into lepton pairs, which restrict $Z^{\prime}$ mass to be slightly heavier than about $4 \mathrm{TeV}[37,38]$.

The other decay mode which has been heavily investigated at the LHC consists in decays into an associated $\ell N_{\ell}$ pair which gives rise to an $\ell \ell j j$ signature. The Majorana nature of the right-handed neutrino allows for probing both the same-sign and opposite-sign dilepton channels [39]. Both the ATLAS and CMS collaborations have looked for such a $W_{R}$ signal, excluding $W_{R}$ masses up to about $4.7 \mathrm{TeV}$ for right-handed (muon or electron) neutrino masses lying between $500 \mathrm{GeV}$ and $2 \mathrm{TeV}[40,41]$. For lower right-handed neutrino masses below $200 \mathrm{GeV}$, which corresponds to our case, the bound is close to $3 \mathrm{TeV}$ (taking into account the suppression from SUSY decay modes) and hence less restrictive than the one originating from dijet searches. For the tau channel, it is even a lot weaker, with $m_{W_{R}}$ excluded at most at $2.9 \mathrm{TeV}$ [42], again without assuming decays to superpartners.

\section{Dark matter constraints}

\section{The no co-annihilation case}

Sneutrino DM mostly annihilates, in LRSUSY scenarios, through an $s$-channel exchange of the $125 \mathrm{GeV}$ Higgs boson $h$. This contrasts with models in which the right-handed sneutrino is a singlet. In this latter case, sneutrino annihilations have indeed to rely either on resonances to reproduce the observed relic abundance [43-46], or on the mixing of left and right sneutrinos [47-50]. In LRSUSY models, righthanded sneutrinos are part of right-handed sleptonic doublets, so that their coupling to the Higgs boson $h$ reads

$$
\lambda_{h \tilde{\nu}_{R} \tilde{\nu}_{R}}=\frac{1}{4} g_{R}^{2} v \sin (\alpha+\beta),
$$

where $g_{R}$ is the $S U(2)_{\mathrm{R}}$ gauge coupling, $v=246 \mathrm{GeV}$ is the SM Higgs VEV, $\alpha$ stands for the mixing angle of the $C P$-even Higgs states and $\tan \beta=v_{2} / v_{1}$. We performed a scan to ascertain the sneutrino mass regions that can produce correct relic density, and present the results in Fig. 1. Our DM relic calculation is performed with MADDM 2.0 [51], and our scan procedure deliberately omits any potential co-annihilation channel (see Sec. III D 2 for the impact of the co-annihilation channels). As detailed below, the particle spectrum and the necessary UFO version [52] of the model have been generated with SARAH 4 [17,53] and SPHENO 3 [54].

The results demonstrate that the structure of the righthanded sneutrino gauge coupling of Eq. (3.6) alone can lead to sufficient annihilations, even further away from the Higgs-funnel resonant region. The horizontal grey band on the left panel of the figure indicates the $2 \sigma$ experimentallyallowed range as derived by the Planck collaboration [8],

$$
\Omega h^{2} \in[0.1163,0.1217],
$$

whilst on the right panel, we report the distribution of the direct detection cross section, $\sigma_{\mathrm{SI}}$, as a function of the sneutrino mass. In this subfigure, we mark as red points those points consistent with the measured relic abundance (as obtained on the left figure). Those points, however, turn out to be ruled out by the best-fit exclusion provided by the 

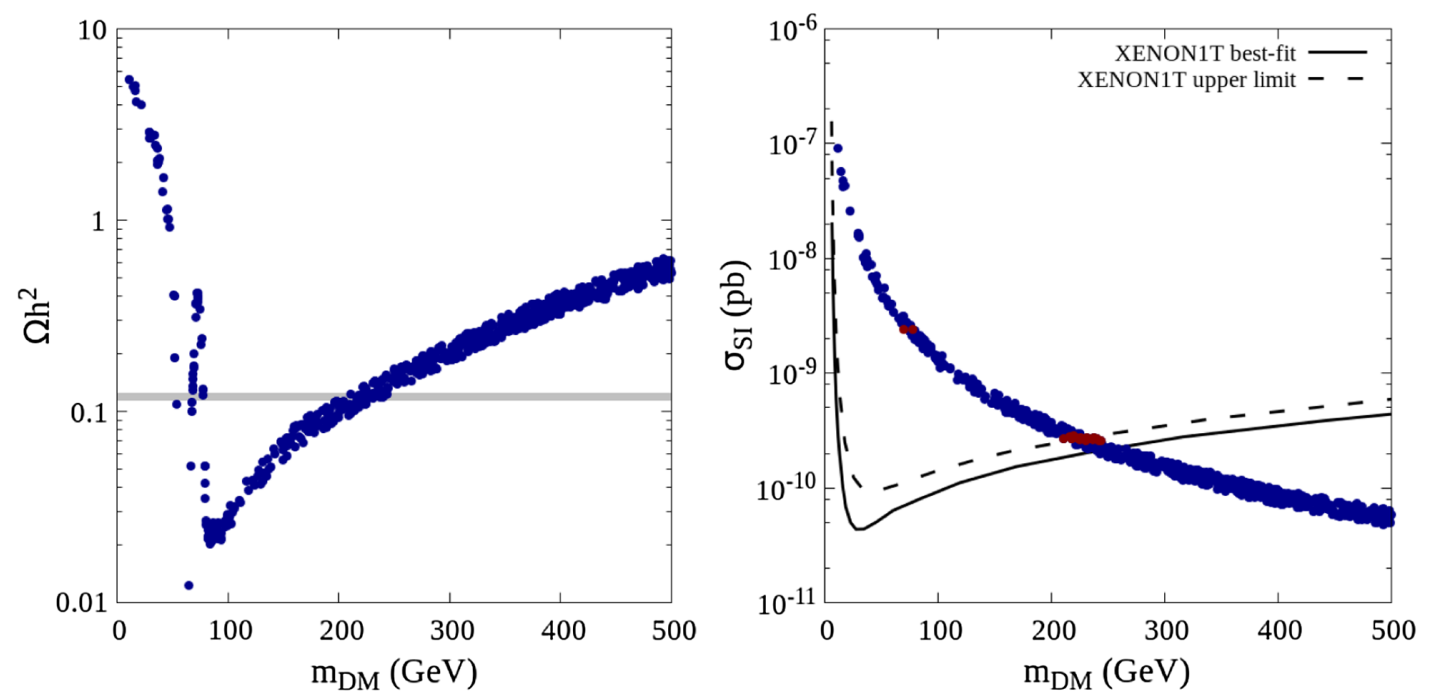

FIG. 1. Dependence of the relic density (left) and direct detection cross section (right) on the right-handed sneutrino mass. We find a viable solution, with respect to present cosmological data, for sneutrino masses around $250 \mathrm{GeV}$. The results do not include DM co-annihilation with any other sparticle.

XENON1T collaboration [23], shown as the solid black line in the figure. We could alternatively rely on the XENON1T $2 \sigma$ upper limit on $\sigma_{\mathrm{SI}}$ presented as a dashed black line, given the large uncertainties that contribute to the cross-section measurements. This leads to the existence of viable configurations that would merit further attention. However, the expected progress in future DM direct detection experiments will challenge these benchmarks, and could potentially exclude the full hypothesis of sneutrino DM in left-right supersymmetry. Direct detection would indeed push for heavier sneutrinos, which turn out to yield over-abundant DM. Including co-annihilations may, however, modify these conclusions.

\section{Co-annihilations}

If there are superpartners that are close in mass to the LSP, they are present when dark matter freezes out and coannihilation processes need to be taken into account [55]. Charginos and neutralinos annihilate more efficiently to SM particles than sneutrinos. Co-annihilations consequently reduce the relic density relative to the no-co-annihilation case, although the effect is Boltzmann-suppressed when the mass difference between the LSP and the co-annihilating particles becomes larger. In this work, we mainly focus on coannihilations of the sneutrino with NLSP neutralino and/or chargino states, LRSUSY models having altogether twelve neutralinos and six singly-charged charginos. Whilst most states are naturally in the multi- $\mathrm{TeV}$ range, some may be lighter and thus relevant from a cosmological standpoint. Their masses are controlled by the soft supersymmetrybreaking parameters for what concerns the gauginos, while the Higgsinos have a mass of the order of $\mu_{\text {eff }}$. Light Higgsinos consist in an appealing option, as there are four neutral and two charged nearly-degenerate bidoublet
Higgsinos that could potentially yield sizeable effects on the relic density.

The bidoublet Higgsinos form a nearly degenerate set of four neutralinos and two charginos and hence co-annihilations are always present if the lightest of these neutralinos is either the LSP, or the NLSP in the case where it is nearly degenerate with the LSP. The Higgsinos co-annihilate mainly via the $\tilde{\chi}_{i}^{0} \tilde{\chi}_{j}^{ \pm} \rightarrow q \bar{q}^{\prime}$ and $\tilde{\chi}_{i}^{0} \tilde{\chi}_{j}^{0} \rightarrow q \bar{q}$ or $V V(V=W, Z)$ channels, processes that are all mediated mainly by $s$-channel $W$ boson, $Z$ boson, and Higgs boson exchanges with the mediator depending on the charges and $C P$ properties of the co-annihilating particles. Annihilations into quarks via gauge boson exchanges are often the dominant channels and the relevant couplings here are standard electroweak gauge couplings.

If a sneutrino LSP is mostly degenerate with the Higgsinos, additional co-annihilations with the sneutrino need to be considered. The most significant of these modes consists in $\tilde{\nu} \tilde{\chi}^{0} \rightarrow \ell^{ \pm} W^{\mp}$ co-annihilations, which proceed via a $t$-channel wino exchange. Since this channel requires either a mixing between the left- and right-handed sneutrinos, or between the left- and right-handed charged winos, both mixings that are small in our model, the corresponding contributions to the relic abundance are relatively small compared to the $\tilde{\nu} \tilde{\nu} \rightarrow V V$ or $t \bar{t}$ modes. For cases in which the splittings between the sneutrino LSP and the lighter neutralinos and charginos are small, it however turns out that neutralino-pair and neutralino-chargino annihilation cross sections are one order of magnitude larger than the sneutrinosneutrino one (provided the Boltzmann suppression is not too important).

One of the benchmark scenarios that will be adopted below yields a relic density that is compatible with Planck data by involving the co-annihilations of a sneutrino LSP 


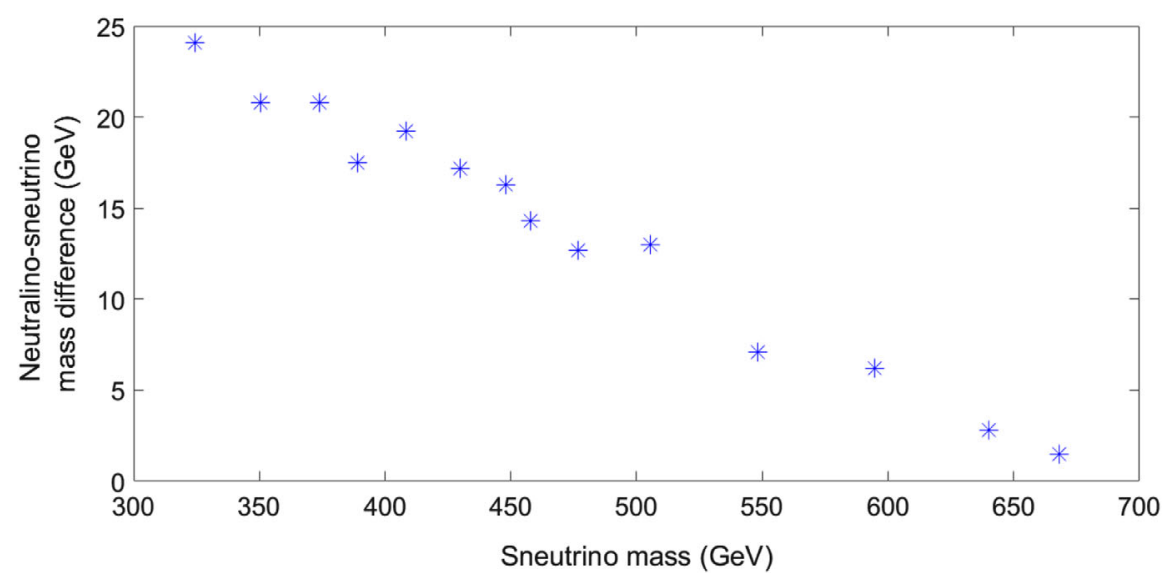

FIG. 2. Distribution of the mass difference between the sneutrino LSP and the NLSP, given as a function of the sneutrino mass. For each point, the relic density matches the Planck value thanks to the bidoublet Higgsino-like neutralinos and charginos that are nearly degenerate and not too much heavier.

with a left-handed wino NLSP. In such a case, the most important (co-)annihilation channels consist of $\tilde{\chi}_{1}^{0} \tilde{\chi}_{1}^{0} \rightarrow$ $W^{+} W^{-}$and $\tilde{\chi}_{1}^{0} \tilde{\chi}_{1}^{ \pm} \rightarrow Z W^{ \pm}$scattering, mediated by $s-$ channel $Z$-boson and $W$-boson exchanges, respectively. As the left-handed wino does not usually mix with any other neutralino or chargino, the effective cross section is then entirely determined by gauge coupling strengths and the Boltzmann suppression stemming from mass difference between the LSP and the NLSP.

The effect of the co-annihilations on a sneutrino LSP density is illustrated in Fig. 2, whilst we refer to the next subsection for the Higgsino LSP case. In Figure 2, we present the dependence of the mass difference between the sneutrino LSP and the NLSP on the sneutrino mass. Each point corresponds to a scenario where the Planck value for the relic density is reproduced as in Eq. (3.7). We observe that cosmologically-viable configurations can be found for mostly any sneutrino mass ranging up to $675 \mathrm{GeV}$, the mass value at which the sneutrino cannot be the LSP anymore. Comparing with the results of Sec. III D 1, the LSP mass can hence be viably shifted by up to several hundreds of $\mathrm{GeV}$ by the sole virtue of the co-annihilation channels.

For many scanned configurations, the co-annihilating new degrees of freedom annihilate less efficiently than the LSP. Their net effect is a reduction of the full annihilation rate at freeze-out instead of an enhancement, so that the relic density is increased [56]. In LRSUSY setups with a sneutrino LSP, this happens either when some of the heavier Higgs bosons are lighter than the LSP, or when the spectrum features nearly degenerate sneutrinos. In addition to the SM-like Higgs boson $h$, LRSUSY spectra indeed always feature MSSM-like Higgs states (namely a $C P$-even Higgs boson $H$, a $C P$-odd Higgs boson $A$ and a charged Higgs boson $H^{ \pm}$) which are nearly degenerate. These can in principle be lighter than the LSP and then impact the relic density in the sneutrino LSP case through $D$-term four-point couplings that drive the $\tilde{\nu}_{R} \tilde{\nu}_{R} \rightarrow H H$,
$A A$ and $H^{+} H^{-}$annihilation channels. Whilst such an option allows for very light sneutrino LSP solutions with respect to the relic abundance, one cannot get a scenario where constraints from direct searches for heavier scalars and flavor physics can simultaneously be satisfied. A light $C P$-odd state indeed sizeably contributes to $B_{s} \rightarrow \mu^{+} \mu^{-}$ [33], which is excluded in the light of current data. Spectra exhibiting several light and degenerate sneutrinos are however not affected by those considerations, so that one may push the sneutrino mass down to about $200 \mathrm{GeV}$. This possibility is, however, ruled out by DM direct detection bounds from XENON1T, as shown in Fig. 1.

The viable parameter space for other types of coannihilating scenarios (featuring light $S U(2)_{\mathrm{R}}$ wino-like or Higgsino-like electroweakinos for instance) are excluded by collider searches for extra gauge bosons or doublycharged Higgs bosons.

\section{Higgsino LSP}

In the previous section, we focused on sneutrino LSP scenarios where co-annihilations with nearly-degenerate bidoublet Higgsinos were yielding the observed DM relic abundance. Conversely, similar cosmologically viable setups could be obtained when the LSP is a neutral Higgsino. In this case, the relic density increases with the LSP mass once all annihilation channels are kinematically open. The Planck value is matched for LSP masses of around $750 \mathrm{GeV}$ when co-annihilations with sneutrinos are ignored. The impact of the latter decreases the effective annihilation cross section and then points towards a slightly lighter LSP of about $675-700 \mathrm{GeV}$. This leads to viable spectra that are fairly heavy, with all lighter states being mostly degenerate bidoublet Higgsinos and sneutrinos. Such a configuration would also be roughly compatible with the AMS-02 results [57], which points to a TeV-scale DM candidate. 
Scenarios in which a gaugino state is nearly degenerate with the Higgsinos do not yield much differences. If the LSP is Higgsino-dominated, with an up to $30 \%$ gaugino admixture, the relic density constraint can be satisfied with slightly lighter LSP masses, and the annihilation channels are nearly the same as in a scenario where only Higgsinos would be co-annihilating. Bino-Higgsino co-annihilations, that could be crucial in the MSSM, do not work in the same way in LRSUSY models. The bino in our model always mixes strongly with the $S U(2)_{\mathrm{R}}$ wino, so that there is no pure bino-state at all. Therefore, if we try to design a scenario in which a bino-state would be degenerate with the Higgsinos, the Higgsinos will also mix with this bino-wino combination. Basically we will end with two states, both admixtures of gauginos and Higgsinos. The mixing lifts the degeneracy among the Higgsinos, so that one state will be lighter and the other heavier than the original with degenerate Higgsinos. This difference with the MSSM is thus completely expected, as the MSSM $\left(U(1)_{\mathrm{Y}}\right)$ bino is here made of an admixture of the neutral $S U(2)_{\mathrm{R}}$ gaugino and the $U(1)_{B-L}$ bino. The well-tempered MSSM scenario consists thus of a triple admixture of states.

However, if the most gaugino-dominated state is the LSP, the mass difference between the co-annihilating particles is larger, so that the net effect on the relic density is Boltzmann-suppressed. The bino-wino mixture does not annihilate as efficiently as Higgsinos, the corresponding relic density turns to be larger than the Planck value, despite the co-annihilations.

\section{BENCHMARKS}

To illustrate our results, we have selected five benchmark points with different dark matter candidates and coannihilation configurations. Each benchmark has a relic density compatible with the Planck results within one standard deviation. For each benchmark, the particle spectrum has been computed at the one-loop level accuracy with SARAH 4 [53] and SPHENo 3.3.8 [54], while the doubly-charged Higgs boson masses have been evaluated with the algorithm described in Ref. [17]. In practice, we first chose $v_{R}$ to yield the $W_{R}$ boson heavy enough. We have then fixed the parameters relevant for satisfying the Higgs sector constraints, namely $\lambda_{R}, v_{S}$ and $\mathbf{h}_{R R}$ (to fix the properties of the doubly-charged Higgs bosons), as well as $\tan \beta$ and $\tan \beta_{R}$ (for masses of the lightest singly-charged, the $C P$-odd and the second $C P$-even Higgs bosons). The correct SM-like Higgs-boson mass is retrieved by adjusting the stop masses and mixings.

For scenarios with a sneutrino LSP, we first set the sneutrino mass to a given value before scanning over the slepton soft masses, $\lambda_{3}$ and $M_{2 L}$. This impacts the NLSP and the other co-annihilating particles as those parameters respectively control the sneutrino, Higgsino and $S U(2)_{\mathrm{L}}$-wino masses. The scan is driven to obtain scenarios featuring a relic density compatible with Planck
TABLE I. Relevant masses of the selected benchmark points.

\begin{tabular}{lrrrrr}
\hline \hline Mass $[\mathrm{GeV}]$ & BP1 & BP2 & \multicolumn{1}{c}{ BP3 } & BP4 & BP5 \\
\hline$m_{W_{R}}$ & 3509.8 & 3509.8 & 3369.5 & 3696.9 & 3369.5 \\
$m_{\tilde{\chi}_{1}^{0}}$ & 608.7 & 696.8 & 405.8 & 429.0 & 690.3 \\
$m_{\tilde{\chi}_{2}^{0}}$ & 707.8 & 716.0 & 417.4 & 665.1 & 718.6 \\
$m_{\tilde{\chi}_{3}^{0}}$ & 712.4 & 716.1 & 417.5 & 665.3 & 718.7 \\
$m_{\tilde{\chi}_{4}^{0}}$ & 712.4 & 717.3 & 419.1 & 666.2 & 719.8 \\
$m_{\tilde{\chi}_{5}^{0}}$ & 713.6 & 851.4 & 704.0 & 677.5 & 768.1 \\
$m_{\tilde{\chi}_{1}^{ \pm}}$ & 699.3 & 705.6 & 411.7 & 429.6 & 712.5 \\
$m_{\tilde{\chi}_{2}^{ \pm}}$ & 711.0 & 715.3 & 416.8 & 663.3 & 717.7 \\
$m_{\tilde{\nu}_{1}}$ & 278.1 & 231.1 & 387.5 & 391.8 & 1066.7 \\
$m_{\tilde{\nu}_{2}}$ & 662.2 & 246.1 & 1092.6 & 658.8 & 1114.2 \\
$m_{\tilde{e}_{1}}$ & 814.6 & 378.5 & 1107.8 & 470.4 & 1150.7 \\
$m_{N_{e}}$ & 136.8 & 137.6 & 131.9 & 122.2 & 131.8 \\
$m_{N_{\mu}}$ & 158.4 & 159.1 & 152.7 & 137.3 & 152.6 \\
$m_{N_{\tau}}$ & 719.6 & 723.2 & 694.9 & 839.4 & 707.6 \\
\hline \hline
\end{tabular}

data. In the co-annihilating Higgsino case, we keep the determination of $\lambda_{3}$ as the last step of the scan. The relevant particle masses for our benchmarks are given in Table I. The most important parameters of the benchmark points are given in Table II. The full spectrum information for all our benchmark scenarios can be obtained from INSPIRE [58].

Tables III and IV show, for all benchmarks, the corresponding branching ratios of the particles relevant for our study. In all cases, the $W_{R}$ boson decays mainly into a dijet system, a lepton and a right-handed neutrino, or into a bidoublet Higgsino-like neutralino-chargino pair, the decays into other electroweakino pairs being smaller or kinematically forbidden. As right-handed neutrinos are Majorana fermions and doublets under $S U(2)_{\mathrm{R}}, W_{R}$ boson decays into a lepton and a right-handed neutrino are similar to its decays into a chargino and neutralino pair, up to phase-space effects. The multiplicity then gives an extra factor of $4 / 3$ in favor of the electroweakino channels. The typical branching fractions of the $W_{R}$ boson are consequently

TABLE II. Values of the most relevant parameters for the selected benchmark points. Here $V^{2}=v_{R}^{2}+v_{R}^{\prime 2}$. All benchmark points share the values $\lambda_{4}=\lambda_{5}=0, \lambda_{L}=0.4, \lambda_{S}=-0.5$ and $\xi_{F}=-5 \times 10^{5} \mathrm{GeV}^{3}$. The full sets of input parameters are available at Ref. [58].

\begin{tabular}{lccccc}
\hline \hline & BP1 & BP2 & BP3 & BP4 & BP5 \\
\hline $\tan \beta$ & 8 & 7 & 7 & 7 & 7 \\
$\tan \beta_{R}$ & 1.05 & 1.045 & 1.045 & 1.04 & 1.045 \\
$V[\mathrm{TeV}]$ & 7.5 & 7.5 & 7.2 & 7.9 & 7.2 \\
$v_{S}[\mathrm{TeV}]$ & 10 & 7.2 & 6.4 & 7.8 & 7.0 \\
$\lambda_{3}$ & 0.10 & 0.14 & 0.0915 & 0.12 & 0.144 \\
$\lambda_{R}$ & 0.85 & 0.90 & 0.90 & 0.90 & 0.90 \\
$M_{1}[\mathrm{GeV}]$ & 400 & 700 & 550 & 750 & 700 \\
$M_{2 L}[\mathrm{GeV}]$ & 900 & 1000 & 900 & 412 & 1200 \\
$M_{2 R}[\mathrm{GeV}]$ & 900 & 1000 & 900 & 1100 & 650 \\
\hline \hline
\end{tabular}


TABLE III. Relevant branching ratios for the BP1 and BP2 benchmark points.

\begin{tabular}{|c|c|c|c|c|c|}
\hline \multicolumn{6}{|c|}{ Branching ratios in the $\mathbf{B P 1}$ scenario } \\
\hline $\operatorname{BR}\left(\tilde{\chi}_{1}^{0} \rightarrow \nu \tilde{\nu}_{1}\right)$ & 1.00 & $\operatorname{BR}\left(\tilde{\chi}_{4}^{0} \rightarrow \nu \tilde{\nu}_{1}\right)$ & 1.00 & $\operatorname{BR}\left(\tilde{e}_{1} \rightarrow e \tilde{\chi}_{1}^{0}\right)$ & 0.96 \\
\hline $\operatorname{BR}\left(\tilde{\chi}_{2}^{0} \rightarrow \nu \tilde{\nu}_{1}\right)$ & 0.04 & $\operatorname{BR}\left(\tilde{\chi}_{5}^{0} \rightarrow \nu \tilde{\nu}_{1}\right)$ & 0.01 & $\operatorname{BR}\left(\tilde{e}_{1} \rightarrow e \tilde{\chi}_{2}^{0}\right)$ & 0.04 \\
\hline $\operatorname{BR}\left(\tilde{\chi}_{2}^{0} \rightarrow Z \tilde{\chi}_{1}^{0}\right)$ & 0.96 & $\operatorname{BR}\left(\tilde{\chi}_{5}^{0} \rightarrow Z \tilde{\chi}_{1}^{0}\right)$ & 0.99 & $\mathrm{BR}\left(W_{R} \rightarrow \tilde{\chi}_{i}^{0} \tilde{\chi}_{j}^{ \pm}\right)$ & 0.23 \\
\hline $\operatorname{BR}\left(\tilde{\chi}_{3}^{0} \rightarrow \nu \tilde{\nu}_{1}\right)$ & 0.98 & $\operatorname{BR}\left(\tilde{\chi}_{1}^{ \pm} \rightarrow W \tilde{\chi}_{1}^{0}\right)$ & 1.00 & $\mathrm{BR}\left(W_{R} \rightarrow N \ell\right)$ & 0.17 \\
\hline $\operatorname{BR}\left(\tilde{\chi}_{3}^{0} \rightarrow Z \tilde{\chi}_{1}^{0}\right)$ & 0.02 & $\operatorname{BR}\left(\tilde{\chi}_{2}^{ \pm} \rightarrow \tau \tilde{\nu}_{1}\right)$ & 1.00 & $\mathrm{BR}\left(W_{R} \rightarrow q q^{\prime}\right)$ & 0.50 \\
\hline \multicolumn{6}{|c|}{ Branching ratios in the $\mathbf{B P 2}$ scenario } \\
\hline $\mathrm{BR}\left(\tilde{\chi}_{1}^{0} \rightarrow \ell \tilde{\ell}\right)$ & 1.00 & $\operatorname{BR}\left(\tilde{\chi}_{5}^{0} \rightarrow W \tilde{\chi}_{1}^{ \pm}\right)$ & 0.11 & $\operatorname{BR}\left(\tilde{\chi}_{2}^{ \pm} \rightarrow \ell \tilde{\nu}_{1}\right)$ & 0.06 \\
\hline $\operatorname{BR}\left(\tilde{\chi}_{2}^{0} \rightarrow \nu \tilde{\nu}_{1}\right)$ & 1.00 & $\operatorname{BR}\left(\tilde{\chi}_{5}^{0} \rightarrow Z \tilde{\chi}_{4}^{0}\right)$ & 0.02 & $\operatorname{BR}\left(\tilde{\chi}_{2}^{ \pm} \rightarrow \tau \tilde{\nu}_{1}\right)$ & 0.93 \\
\hline $\operatorname{BR}\left(\tilde{\chi}_{3}^{0} \rightarrow \nu \tilde{\nu}_{1}\right)$ & 1.00 & $\operatorname{BR}\left(\tilde{\chi}_{5}^{0} \rightarrow h \tilde{\chi}_{1}^{0}\right)$ & 0.03 & $\operatorname{BR}\left(\tilde{e}_{1} \rightarrow q q \tilde{\nu}_{1,2}\right)$ & 1.00 \\
\hline $\operatorname{BR}\left(\tilde{\chi}_{4}^{0} \rightarrow \nu \tilde{\nu}_{1}\right)$ & 0.69 & $\operatorname{BR}\left(\tilde{\chi}_{1}^{ \pm} \rightarrow N \tilde{e}_{1}\right)$ & 0.32 & $\mathrm{BR}\left(W_{R} \rightarrow \tilde{\chi}_{i}^{0} \tilde{\chi}_{j}^{ \pm}\right)$ & 0.22 \\
\hline $\operatorname{BR}\left(\tilde{\chi}_{4}^{0} \rightarrow \ell \tilde{\ell}\right)$ & 0.30 & $\operatorname{BR}\left(\tilde{\chi}_{1}^{ \pm} \rightarrow \tau \tilde{\nu}_{1}\right)$ & 0.62 & $\mathrm{BR}\left(W_{R} \rightarrow N \ell\right)$ & 0.16 \\
\hline $\operatorname{BR}\left(\tilde{\chi}_{4}^{0} \rightarrow \ell \bar{\ell} \tilde{\chi}_{1}^{0}\right)$ & 0.01 & $\operatorname{BR}\left(\tilde{\chi}_{1}^{ \pm} \rightarrow \ell \bar{\nu}_{\ell}^{\prime} \tilde{\chi}_{1}^{0}\right)$ & 0.03 & $\mathrm{BR}\left(W_{R} \rightarrow q q^{\prime}\right)$ & 0.50 \\
\hline $\operatorname{BR}\left(\tilde{\chi}_{5}^{0} \rightarrow \ell \tilde{\ell}\right)$ & 0.83 & $\operatorname{BR}\left(\tilde{\chi}_{1}^{ \pm} \rightarrow q \bar{q}^{\prime} \tilde{\chi}_{1}^{0}\right)$ & 0.03 & & \\
\hline
\end{tabular}

TABLE IV. Relevant branching ratios for the BP3, BP4 and BP5 benchmark points.

\begin{tabular}{|c|c|c|c|c|c|}
\hline \multicolumn{6}{|c|}{ Branching ratios in the $\mathbf{B P 3}$ scenario } \\
\hline $\operatorname{BR}\left(\tilde{\chi}_{1}^{0} \rightarrow \nu \tilde{\nu}_{1}\right)$ & 1.00 & $\operatorname{BR}\left(\tilde{\chi}_{5}^{0} \rightarrow h \tilde{\chi}_{1}^{0}\right)$ & 0.21 & $\operatorname{BR}\left(\tilde{e}_{1} \rightarrow e \tilde{\chi}_{1}^{0}\right)$ & 0.03 \\
\hline $\operatorname{BR}\left(\tilde{\chi}_{2}^{0} \rightarrow \nu \tilde{\nu}_{1}\right)$ & 1.00 & $\operatorname{BR}\left(\tilde{\chi}_{1}^{ \pm} \rightarrow \tau \tilde{\nu}_{1}\right)$ & 0.85 & $\operatorname{BR}\left(\tilde{e}_{1} \rightarrow e \tilde{\chi}_{5}^{0}\right)$ & 0.97 \\
\hline $\operatorname{BR}\left(\tilde{\chi}_{3}^{0} \rightarrow \nu \tilde{\nu}_{1}\right)$ & 1.00 & $\operatorname{BR}\left(\tilde{\chi}_{1}^{ \pm} \rightarrow \ell \bar{\nu}_{\ell}^{\prime} \tilde{\chi}_{1}^{0}\right)$ & 0.05 & $\mathrm{BR}\left(W_{R} \rightarrow \tilde{\chi}_{i}^{0} \tilde{\chi}_{j}^{ \pm}\right)$ & 0.22 \\
\hline $\operatorname{BR}\left(\tilde{\chi}_{4}^{0} \rightarrow \nu \tilde{\nu}_{1}\right)$ & 1.00 & $\mathrm{BR}\left(\tilde{\chi}_{1}^{ \pm} \rightarrow q \bar{q}^{\prime} \tilde{\chi}_{1}^{0}\right)$ & 0.10 & $\mathrm{BR}\left(W_{R} \rightarrow N \ell\right)$ & 0.17 \\
\hline $\operatorname{BR}\left(\tilde{\chi}_{5}^{0} \rightarrow Z \tilde{\chi}_{1}^{0}\right)$ & 0.01 & $\operatorname{BR}\left(\tilde{\chi}_{2}^{ \pm} \rightarrow \tau \tilde{\nu}_{1}\right)$ & 1.00 & $\mathrm{BR}\left(W_{R} \rightarrow q q^{\prime}\right)$ & 0.51 \\
\hline $\operatorname{BR}\left(\tilde{\chi}_{5}^{0} \rightarrow Z \tilde{\chi}_{4}^{0}\right)$ & 0.21 & & & & \\
\hline \multicolumn{6}{|c|}{ Branching ratios in the $\mathbf{B P 4}$ scenario } \\
\hline $\operatorname{BR}\left(\tilde{\chi}_{1}^{0} \rightarrow \nu \tilde{\nu}_{1}\right)$ & 1.00 & $\operatorname{BR}\left(\tilde{\chi}_{4}^{0} \rightarrow Z \tilde{\chi}_{1}^{0}\right)$ & 0.32 & $\operatorname{BR}\left(\tilde{\chi}_{2}^{ \pm} \rightarrow Z \tilde{\chi}_{1}^{ \pm}\right)$ & 0.10 \\
\hline $\operatorname{BR}\left(\tilde{\chi}_{2}^{0} \rightarrow Z \tilde{\chi}_{1}^{0}\right)$ & 0.32 & $\mathrm{BR}\left(\tilde{\chi}_{5}^{0} \rightarrow W \tilde{\chi}_{1}^{ \pm}\right)$ & 0.65 & $\operatorname{BR}\left(\tilde{\chi}_{2}^{ \pm} \rightarrow q \bar{q}^{\prime} \tilde{\chi}_{1}^{0}\right)$ & 0.17 \\
\hline $\operatorname{BR}\left(\tilde{\chi}_{2}^{0} \rightarrow W \tilde{\chi}_{1}^{ \pm}\right)$ & 0.68 & $\operatorname{BR}\left(\tilde{\chi}_{5}^{0} \rightarrow Z \tilde{\chi}_{1}^{0}\right)$ & 0.01 & $\mathrm{BR}\left(\tilde{\chi}_{2}^{ \pm} \rightarrow q \bar{q} \tilde{\chi}_{1}^{ \pm}\right)$ & 0.10 \\
\hline $\operatorname{BR}\left(\tilde{\chi}_{3}^{0} \rightarrow Z \tilde{\chi}_{1}^{0}\right)$ & 0.01 & $\operatorname{BR}\left(\tilde{\chi}_{5}^{0} \rightarrow h \tilde{\chi}_{1}^{0}\right)$ & 0.23 & $\operatorname{BR}\left(\tilde{\chi}_{2}^{ \pm} \rightarrow \ell \bar{\ell}_{\chi}^{ \pm}\right)$ & 0.09 \\
\hline $\operatorname{BR}\left(\tilde{\chi}_{3}^{0} \rightarrow h \tilde{\chi}_{1}^{0}\right)$ & 0.32 & $\operatorname{BR}\left(\tilde{\chi}_{5}^{0} \rightarrow \ell \bar{\ell} \tilde{\chi}_{1}^{0}\right)$ & 0.02 & $\operatorname{BR}\left(\tilde{\chi}_{2}^{ \pm} \rightarrow \nu \bar{\nu} \tilde{\chi}_{1}^{ \pm}\right)$ & 0.07 \\
\hline $\operatorname{BR}\left(\tilde{\chi}_{3}^{0} \rightarrow W \tilde{\chi}_{1}^{ \pm}\right)$ & 0.34 & $\mathrm{BR}\left(\tilde{\chi}_{5}^{0} \rightarrow q \bar{q} \tilde{\chi}_{1}^{0}\right)$ & 0.09 & $\operatorname{BR}\left(\tilde{e}_{1} \rightarrow e \tilde{\chi}_{1}^{0}\right)$ & 1.00 \\
\hline $\operatorname{BR}\left(\tilde{\chi}_{3}^{0} \rightarrow \ell \bar{\ell} \tilde{\chi}_{1}^{0}\right)$ & 0.02 & $\operatorname{BR}\left(\tilde{\chi}_{1}^{ \pm} \rightarrow \ell \tilde{\nu}_{1}\right)$ & 1.00 & $\operatorname{BR}\left(W_{R} \rightarrow \tilde{\chi}_{i}^{0} \tilde{\chi}_{j}^{ \pm}\right)$ & 0.22 \\
\hline $\operatorname{BR}\left(\tilde{\chi}_{3}^{0} \rightarrow q \bar{q} \tilde{\chi}_{1}^{0}\right)$ & 0.28 & $\operatorname{BR}\left(\tilde{\chi}_{2}^{ \pm} \rightarrow \ell \tilde{\nu}_{1}\right)$ & 0.18 & $\mathrm{BR}\left(W_{R} \rightarrow N \ell\right)$ & 0.16 \\
\hline $\operatorname{BR}\left(\tilde{\chi}_{4}^{0} \rightarrow W \tilde{\chi}_{1}^{ \pm}\right)$ & 0.67 & $\operatorname{BR}\left(\tilde{\chi}_{2}^{ \pm} \rightarrow W \tilde{\chi}_{1}^{0}\right)$ & 0.27 & $\mathrm{BR}\left(W_{R} \rightarrow q q^{\prime}\right)$ & 0.50 \\
\hline \multicolumn{6}{|c|}{ Branching ratios in the BP5 scenario } \\
\hline $\operatorname{BR}\left(\tilde{\chi}_{2}^{0} \rightarrow \ell \bar{\ell} \tilde{\chi}_{1}^{0}\right)$ & 0.11 & $\operatorname{BR}\left(\tilde{\chi}_{5}^{0} \rightarrow q q^{\prime} \tilde{\chi}_{1}^{ \pm}\right)$ & 0.56 & $\operatorname{BR}\left(\tilde{\chi}_{2}^{ \pm} \rightarrow \ell \bar{\nu}_{\ell}^{\prime} \tilde{\chi}_{1}^{0}\right)$ & 0.33 \\
\hline $\operatorname{BR}\left(\tilde{\chi}_{2}^{0} \rightarrow \nu \bar{\nu} \tilde{\chi}_{1}^{0}\right)$ & 0.21 & $\operatorname{BR}\left(\tilde{\chi}_{5}^{0} \rightarrow \ell \nu_{\ell}^{\prime} \tilde{\chi}_{1}^{ \pm}\right)$ & 0.30 & $\operatorname{BR}\left(\tilde{e}_{1} \rightarrow e \tilde{\chi}_{1}^{0}\right)$ & 0.32 \\
\hline $\operatorname{BR}\left(\tilde{\chi}_{2}^{0} \rightarrow q \bar{q} \tilde{\chi}_{1}^{0}\right)$ & 0.68 & $\operatorname{BR}\left(\tilde{\chi}_{5}^{0} \rightarrow q \bar{q} \tilde{\chi}_{4}^{0}\right)$ & 0.10 & $\operatorname{BR}\left(\tilde{e}_{1} \rightarrow e \tilde{\chi}_{5}^{0}\right)$ & 0.68 \\
\hline $\operatorname{BR}\left(\tilde{\chi}_{3}^{0} \rightarrow q \bar{q} \tilde{\chi}_{1}^{0}\right)$ & 1.00 & $\operatorname{BR}\left(\tilde{\chi}_{5}^{0} \rightarrow \ell \bar{\ell}^{2} \tilde{\chi}_{4}^{0}\right)$ & 0.04 & $\mathrm{BR}\left(W_{R} \rightarrow \tilde{\chi}_{i}^{0} \tilde{\chi}_{j}^{ \pm}\right)$ & 0.22 \\
\hline $\operatorname{BR}\left(\tilde{\chi}_{4}^{0} \rightarrow \ell \bar{\ell} \tilde{\chi}_{1}^{0}\right)$ & 0.11 & $\operatorname{BR}\left(\tilde{\chi}_{1}^{ \pm} \rightarrow \ell \bar{\nu}_{\ell}^{\prime} \tilde{\chi}_{1}^{0}\right)$ & 0.34 & $\mathrm{BR}\left(W_{R} \rightarrow N \ell\right)$ & 0.17 \\
\hline $\operatorname{BR}\left(\tilde{\chi}_{4}^{0} \rightarrow \nu \bar{\nu} \tilde{\chi}_{1}^{0}\right)$ & 0.21 & $\operatorname{BR}\left(\tilde{\chi}_{1}^{ \pm} \rightarrow q \bar{q}^{\prime} \tilde{\chi}_{1}^{0}\right)$ & 0.66 & $\mathrm{BR}\left(W_{R} \rightarrow q q^{\prime}\right)$ & 0.52 \\
\hline $\mathrm{BR}\left(\tilde{\chi}_{3}^{0} \rightarrow q \bar{q} \tilde{\chi}_{1}^{0}\right)$ & 0.68 & $\mathrm{BR}\left(\tilde{\chi}_{2}^{ \pm} \rightarrow q \bar{q}^{\prime} \tilde{\chi}_{1}^{0}\right)$ & 0.67 & & \\
\hline
\end{tabular}




$$
\begin{aligned}
\operatorname{BR}\left(W_{R} \rightarrow j j\right) & \sim 50 \%, \\
\operatorname{BR}\left(W_{R} \rightarrow N \ell\right) & \sim 16 \%, \\
\operatorname{BR}\left(W_{R} \rightarrow \tilde{\chi} \tilde{\chi}\right) & \sim 22 \%,
\end{aligned}
$$

with subdominant channels into various combinations of slepton pairs $(\sim 4 \%)$ or gauge and Higgs bosons $(\sim 4 \%)$. In all the setups except in our second benchmark scenario BP2, the lightest neutralino is either stable or decays invisibly into a neutrino and a sneutrino LSP. $W_{R}$ decays hence often lead to a significant amount of missing transverse energy, which provide clear handles on LRSUSY.

\section{A. The BP1 scenario}

In this benchmark scenario, that is very close to the second scenario introduced in Ref. [18], the LSP is a righthanded tau sneutrino. It is much lighter than any other superpartner, so that the relic density matches the Planck value only by virtue of the sole sneutrino-pair annihilations. The sneutrino mass lies in the $250-300 \mathrm{GeV}$ mass window, in agreement with the results of Sec. III D 1, and the $\mathbf{h}_{R R}$ Yukawa texture pushes the tau sneutrino to be the lightest one with a mass of $278 \mathrm{GeV}$. The lightest neutralino is an admixture of the $\tilde{B}$ and $\tilde{W}_{R}^{0}$ gauginos and has a mass close to $600 \mathrm{GeV}$, while the next-to-lightest (charged and neutral) electroweakinos are bidoublet-like Higgsinos, with masses around $700 \mathrm{GeV}$.

In this scenario, the preferred $W_{R}$ boson supersymmetric decay modes involve $\tilde{\chi}_{3,4}^{0} \tilde{\chi}_{1}^{ \pm}$and $\tilde{\chi}_{2,5}^{0} \tilde{\chi}_{2}^{ \pm}$final states, each with a branching ratio of about $5 \%$. The three $\tilde{\chi}_{1,3,4}^{0}$ neutralinos decay almost completely invisibly to a $\nu \tilde{\nu}$ pair, although the $\tilde{\chi}_{3}^{0}$ state subdominantly decays visibly into a $Z \tilde{\chi}_{1}^{0}$ pair (with a branching ratio of $2 \%$ ). This $Z \tilde{\chi}_{1}^{0}$ decay mode is also the main decay channel for the $\tilde{\chi}_{2,5}^{0}$ states. With branching ratios greater than $95 \%$, this can further give rise to the production of opposite-sign same-flavor lepton pairs at colliders. The two lighter charginos respectively decay into $W^{ \pm} \tilde{\chi}_{1}^{0}$ and $\tau^{ \pm} \tilde{\nu}$ systems, the lepton flavor in the latter case being connected to the LSP nature. Altogether such a spectrum has a good chance to copiously produce hard leptons at colliders, in association with missing energy, which could provide handles on the model.

\section{B. The BP2 scenario}

Our second benchmark features two light right-handed sneutrinos, of the tau and electron flavors, so that the reproduction of the right relic density value largely relies on co-annihilations. As mentioned in Sec. III D 2, the LSP mass is lower than in setups like the one of our benchmark BP1, where co-annihilations are negligible. The lighter electroweakinos, being of a bidoublet Higgsino nature, are heavier with a mass close to $700 \mathrm{GeV}$, whilst the lightest charged slepton is a right-handed selectron with a mass of $378 \mathrm{GeV}$. In addition, the soft mass configuration yielding the sneutrino hierarchy additionally makes the stau states heavier.

Here, the supersymmetric $W_{R}$ boson decays mainly involve $\tilde{\chi}_{1,4}^{0} \tilde{\chi}_{2}^{ \pm}$and $\tilde{\chi}_{2,3}^{0} \tilde{\chi}_{1}^{ \pm}$final states, each with a branching fraction close to $5 \%$. The lightest neutralino always decays into an $e^{ \pm} \tilde{e}_{R}^{\mp}$ system, which is also a relatively dominant decay mode of the $\tilde{\chi}_{4}^{0}$ state with a branching ratio of $30 \%$ (muonic contributions being subdominant). In contrast, the $\tilde{\chi}_{2,3}^{0}$ neutralinos decay invisibly. Because of the structure of the Yukawa couplings, the Higgsino-like charginos decay mostly into $\tau \tilde{\nu}$ systems, although $\tilde{\chi}_{1}^{ \pm}$decay into a $N_{e} \tilde{e}_{R}^{ \pm}$final state is significant too. Finally, the selectrons often appearing at the end of the decay chain dominantly decay via a virtual $W_{R}$ boson into a $j j \tilde{\nu}_{e}$ system.

This benchmark point can be probed at colliders through a signature involving multilepton final states, the corresponding rate being large enough. The signal often contains electrons, by the nature of the spectrum featuring light electron sneutrino and selectron. Tau leptons are also largely produced, in particular when decays involve charginos $\left(\tilde{\chi}_{1 / 2}^{ \pm}\right)$.

\section{The BP3 scenario}

For our third benchmark, we picked a scenario where the LSP is a right-handed tau sneutrino almost degenerate with a set of bidoublet Higgsinos. Contrary to the BP2 scenario, the LSP is moderately heavier with a mass of $387 \mathrm{GeV}$, and the right relic density is once again obtained thanks to coannihilations. The Higgsinos are about $20-35 \mathrm{GeV}$ heavier.

The supersymmetric decays of the $W_{R}$ boson are similar to the previous cases. The main electroweakino channels, with a branching ratio of $5.5 \%$ each, involve $\tilde{\chi}_{1,4}^{0} \tilde{\chi}_{2}^{ \pm}$and $\tilde{\chi}_{2,3}^{0} \tilde{\chi}_{1}^{ \pm}$systems. The four lighter neutralinos all decay invisibly into a sneutrino/neutrino pair, and the two lighter charginos decay mostly into $\tau \tilde{\nu}$ systems by virtue of the large tau Yukawa coupling, the other channels being threebody and involving virtual $W$ bosons. Although the decays of heavier neutralinos are visible, they are barely produced via intermediate $S U(2)_{\mathrm{R}}$ gauge bosons. This scenario hence manifests itself at colliders through an enhanced production of tau leptons in association with missing transverse energy.

\section{The BP4 scenario}

Our fourth scenario has very different features from the previous one, although both have a sneutrino LSP giving rise to the right relic abundance through co-annihilations. The latter, however, involves this time $S U(2)_{\mathrm{L}}$ wino-like neutralinos and charginos, and the LSP is here an electron sneutrino. This means that the associated collider signature involves electrons instead of taus. The LSP sneutrino mass is of $391 \mathrm{GeV}$, and the lighter neutralinos and charginos have masses of about $430 \mathrm{GeV}$. This mass difference, 
slightly larger than for the BP3 scenario, is necessary for yielding a relic density matching the Planck results, because of the existence of additional DM annihilation subprocesses into right-handed neutrinos via $t$-channel neutralino exchanges. As will be shown below, this annihilation channel is crucial for DM indirect detection. Finally, the bidoublet Higgsinos are heavier, with masses lying around $740 \mathrm{GeV}$.

The neutral and charged light winos decay into $\nu \tilde{\nu}$ and $e \tilde{\nu}$ pairs, respectively, as those are the only possible decay modes. The heavier neutralinos and charginos feature in addition significant branching ratios into the $W \tilde{\chi}_{1}$ and $Z \tilde{\chi}_{1}$ modes, so that $W_{R}$ boson production and supersymmetric decay into electroweakinos (with branching fractions similar to the other benchmarks) could lead to an important production of hard jets and leptons, and electrons in particular, at colliders. Representative signatures of this benchmark feature the intermediate presence of weak bosons whose reconstruction could provide interesting handles to unravel the signals.

\section{E. The BP5 scenario}

In contrast to all other scenarios, the lighter superpartners are all bidoublet Higgsinos and the numerous existing coannihilation modes allow for a viable neutralino DM candidate with a mass of about $700 \mathrm{GeV}$. This rather heavy spectrum consists in a perfect example of stealth supersymmetry. As any new gauge boson or colored superpartner is heavy enough for their production rate to be suppressed, any potential collider signal becomes hard to get. Even when considering cascades such as those originating from the production of a single $W_{R}$ boson, a large integrated luminosity would be necessary to observe any signal. The resulting rate is reasonable enough to give hope for detection.

The lightest neutralinos and charginos being nearly degenerate, their decay proceed via three-body channels. The $\tilde{\chi}_{2,4}^{0}$ states hence give rise to $\nu \bar{\nu} \tilde{\chi}_{1}^{0}(21 \%), \ell^{+} \ell^{-} \tilde{\chi}_{1}^{0}$ $(11 \%)$ and $j j \tilde{\chi}_{1}^{0}(68 \%)$ final states through an off-shell $Z$ boson, whilst the $\tilde{\chi}_{3}^{0}$ neutralino decays in contrast dominantly through an off-shell $h$ boson into a $b \bar{b} \tilde{\chi}_{1}^{0}$ final state. Similarly, chargino decays involve a virtual charged $W$ boson instead. The main $W_{R}$ boson signature hence consists in a production of numerous leptons, jets and missing energy.

\section{IMPLICATIONS FOR DARK MATTER INDIRECT DETECTION}

In this section we discuss the implications of indirect DM searches on the representative LRSUSY scenarios reproducing the Planck results introduced in the previous section. Since the center of the Milky Way and dwarf spheroidal galaxies (dSPhs) are enriched in dark matter, various indirect searches for DM focus on these regions of the universe to extract a DM signal using various classes of cosmic rays, see e.g., Refs. [59-61]. Calculations associated with neutral particles (i.e., photons and neutrinos) do not suffer from propagation uncertainties, so that gamma rays or neutrino fluxes can be efficiently used to probe DM annihilation. In particular, gamma-ray flux measurements are widely considered to constrain DM annihilation into varied SM states, thanks to the ease of their detection [59]. Constraints on specific final states have been recently evaluated by the Fermi collaboration, using both the continuum gamma-ray fluxes originating from dSPhs [62] or from the galactic center [63]. In addition, the implications on DM annihilation into new physics final states like right-handed neutrinos have also been studied [64].

Constraints on the late-time thermally-averaged DM annihilation cross section $(\langle\sigma v\rangle)$ put forward by the Fermi experiment all assume that DM annihilates into a particular SM channel. However, several annihilation channels are generally open, depending on the model itself and on the exact value of its free parameters. Furthermore, new physics annihilation modes can be open too, like in LRSUSY scenarios where DM often annihilates into righthanded neutrinos. For illustrative purposes, we start by estimating the prompt gamma-ray flux originating from DM annihilation in the context of our characteristic benchmark scenario BP4, where DM annihilation into rightneutrinos is possible along with multiple other channels. We next attempt to put an upper bound on the DM annihilation cross section from the observation by Fermi-LAT of the gamma-ray spectrum issued from 15 $\mathrm{dSPh}$, in the $0.5-500 \mathrm{GeV}$ energy range [62]. We ignore any potential constraints that could emerge from measurements of the gamma-ray flux originating from the galactic center, as the expectation is comparable but plagued by a large background stemming from other astrophysical processes [63].

The observed gamma-ray flux $\left(\Phi_{\gamma}\right)$ is related to the DM annihilation cross section as

$\frac{\mathrm{d} \Phi_{\gamma}}{\mathrm{d} E}\left(E_{\gamma}, \Delta \Omega\right)=\frac{1}{4 \pi} \frac{\langle\sigma v\rangle}{2 m_{\mathrm{DM}}^{2}} \frac{\mathrm{d} N_{\gamma}}{\mathrm{d} E_{\gamma}} \int \mathrm{d} \Omega \int_{\text {l.o.s }} \mathrm{d} r \rho^{2}(r)$,

where the differential gamma-ray flux embedded in a solid angle $\Delta \Omega$ has been equated to the prompt gamma-ray flux generated from the annihilation of a pair of DM particles of mass $m_{\mathrm{DM}}$. The prefactor of the integral includes the thermally-averaged DM annihilation cross section $\langle\sigma v\rangle$ today and in the relevant galaxies, and the differential number density of photons within an energy bin of size $\mathrm{d} E$, $\mathrm{d} N_{\gamma} / \mathrm{d} E_{\gamma}$. The two integrals in the right-hand side represent the so-called astrophysical $J$-factor and account for the squared density of dark matter $\rho$ along the line of sight (l.o.s). We have relied on NFW profiles to estimate the $J$ factor $[62,65]$, which corresponds to an uncertainty of at 

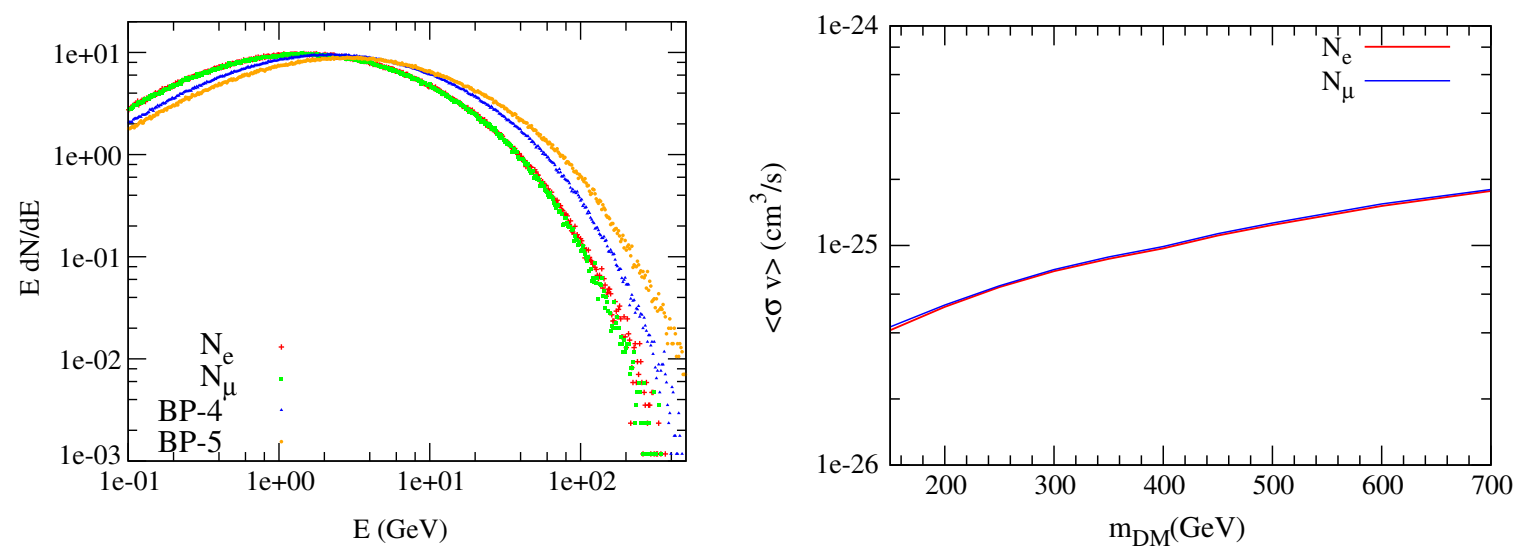

FIG. 3. Photon number density spectrum (left) as derived from the primary gamma-ray flux originating from DM annihilation into right-handed neutrinos, shown as a function of energy. Results are presented for a showcase scenario in which $m_{\mathrm{DM}}=400 \mathrm{GeV}$ and for electron (red) and muon (green) neutrinos of $130 \mathrm{GeV}$, as well as for both the BP4 (blue) and BP5 (orange) scenarios. The upper limits on the DM annihilation cross-section at the 95\% confidence level, derived from Fermi dSPhs data, are also shown for both the righthanded electron and muon channels as a function of the DM mass (right).

most $30 \%$ [62]. Accounting for a different density profile is however not expected to significantly affect the results [62]. The estimation of the contribution from the inverse Compton effect is plagued by a different uncertainty, most notably the uncertainty in the diffusion coefficient, which can affect the final photon spectrum up to one order of magnitude [66]. As we solely consider prompt gamma-ray production at the source, any other contribution such as those stemming from inverse Compton scattering, bremsstrahlung and synchrotron radiation are neglected. While the latter two are subdominant, inverse Compton scattering at dwarf galaxies is potentially relevant but necessitates a detailed modelling of electron and positron propagation to the observer. It will therefore be omitted from our calculations.

In the left panel of Figure 3, we present the (normalized) prompt gamma-ray flux produced by the annihilation of pairs of DM particles into electronic and muonic righthanded neutrinos $N_{e}$ (red) and $N_{\mu}$ (green), for a DM mass of $m_{\mathrm{DM}}=400 \mathrm{GeV}$ and neutrino masses of $130 \mathrm{GeV}$. The particle shower generated from the neutrino decays,

$$
N_{e} \rightarrow e^{ \pm} q q^{\prime} \quad \text { and } \quad N_{\mu} \rightarrow \mu^{ \pm} q q^{\prime}
$$

has been simulated with PyTHIA 8 [67]. In addition, we have also shown results for the BP4 (blue) and BP5 (orange) representative benchmark scenarios featuring masses in the same ball park. In the right panel of the figure, we derive $95 \%$ confidence level limits on the dark matter annihilation cross section in neutrinos from the Fermi results, making use of the publicly available likelihood calculator GAMLiKE [65]. We then apply these findings to our BP4 scenario. From all the competing subprocesses, the driving component turns out to be the annihilation of sneutrinos in a pair of $W$ bosons $(\sim 45 \%)$, and $Z$ bosons $(\sim 21 \%)$, as well as into Higgs bosons $(\sim 15 \%)$, followed by right-handed neutrinos $(\sim 10 \%)$ and top quarks $(\sim 8 \%)$. The corresponding annihilation cross section at present time being $4.3 \times 10^{-27} \mathrm{~cm}^{3} / \mathrm{s}$, the scenario turns out to be safe relative to Fermi observations that exclude, at the $95 \%$ confidence level, cross sections of $9.0 \times 10^{-26} \mathrm{~cm}^{3} / \mathrm{s}$.

We obtain a similar conclusion for our other benchmark scenarios with a similar dark matter mass. In the BP5 scenario with a slightly heavier DM candidate of $690 \mathrm{GeV}$, we get a slightly harder gamma-ray spectrum (left panel of Fig. 3), the dominant process driving the results being the annihilation of pairs of neutralinos into charged $(\sim 45 \%)$ and neutral $(\sim 33 \%)$ electroweak bosons, as well as into $h Z$ associated pairs $(\sim 12 \%)$. The corresponding thermallyaveraged annihilation cross section at the present epoch reads $1.60 \times 10^{-28} \mathrm{~cm}^{3} / \mathrm{s}$, which is again well below the computed Fermi exclusion of $1.7 \times 10^{-25} \mathrm{~cm}^{3} / \mathrm{s}$.

\section{RESONANT PRODUCTION OF CHARGINOS AND NEUTRALINOS AT COLLIDERS}

\section{A. Generalities and analysis definition}

A robust experimental confirmation of the existence of a left-right symmetry would incontrovertibly consist in the discovery of a charged $S U(2)_{\mathrm{R}}$ gauge boson $W_{R}$. The ATLAS and CMS collaborations have therefore extensively searched for varied $W_{R}$ boson signals, in particular in the $\ell \ell j j$ channel. $W_{R}$ boson masses ranging up to about 4.5 TeV have been excluded, assuming that at least one of the right-handed neutrinos $N$ is lighter than the new boson [40], the exact value of the bound being subjected to the $N$ mass. More precisely, there is no constraint when $m_{N} \simeq m_{W_{R}}$ or when $m_{N}$ is below a certain threshold, (e.g., the $m_{W_{R}}>3 \mathrm{TeV}$ region is unconstrained if $m_{N} \lesssim 150 \mathrm{GeV}$ ) [40]. In this case, much more robust 
TABLE V. Definition of the signal regions (SR) of the CMS analysis of Ref. [19] that we use as potentially best probes of our cosmologically-favored LRSUSY scenarios.

\begin{tabular}{lc}
\hline \hline SR & Requirements \\
\hline A44 & $N_{\ell}=3, N_{\tau}=0, N_{\mathrm{OSSF}} \geq 1, M_{T}>160 \mathrm{GeV}, E_{T} \geq 200 \mathrm{GeV}, M_{\ell \ell} \geq 105 \mathrm{GeV}$ \\
C18 & $N_{\ell}=2, N_{\tau}=1, N_{\mathrm{OSSF}}=1, M_{T 2}>100 \mathrm{GeV}, E_{T} \geq 200 \mathrm{GeV}, M_{\ell \ell} \geq 105 \mathrm{GeV}$ \\
D16 & $N_{\ell}=2, N_{\tau}=1, N_{\mathrm{OS}}=1, N_{\mathrm{SF}}=0, M_{T 2}>100 \mathrm{GeV}, E_{T} \geq 200 \mathrm{GeV}$ \\
G05 & $N_{\ell} \geq 4, N_{\tau}=0, N_{\mathrm{OSSF}} \geq 2, E_{T} \geq 200 \mathrm{GeV}$ \\
H04 & $N_{\ell} \geq 4, N_{\tau}=0, N_{\mathrm{OSSF}}<2, E_{T} \geq 150 \mathrm{GeV}$ \\
\hline \hline
\end{tabular}

bounds, $m_{W_{R}} \gtrsim 3.5 \mathrm{TeV}$, are obtained from the analysis of dijet probes for extra gauge bosons [35,36]. All these bounds can, however, be relaxed in an LRSUSY context, thanks to the presence of additional (supersymmetric) decay modes of the $W_{R}$ boson.

In the scenarios investigated in this work, we accommodate a correct DM relic density by enforcing the presence of multiple neutralino and chargino states that are slightly heavier than the LSP. As described in Sec. IV, this yields to new possible decay channels for the $W_{R}$ boson, associated with a combined branching ratio that could be as large as $25 \%$. This opens up the opportunity to look for a $W_{R}$ boson through typical electroweakino searches targeting multileptonic final states exhibiting a large amount of missing energy $E_{T}$. As such signals are absent in minimal nonsupersymmetric left-right extensions of the SM, they consist, together with the would-be observation of a $W_{R}$ boson, of clear evidence for LRSUSY. The existence of these new $W_{R}$ boson signals is also responsible for the reduction of the reach of the classical $W_{R}$ boson searches, as the branching ratios relevant for the latter are automatically reduced by virtue of the new decay modes. Moreover, these new channels, even if featuring missing energy, also provide the opportunity to reconstruct a $W_{R}$ boson mass from the kinematic thresholds featured by numerous transverse variables.

To illustrate this point, we have followed the CMS multilepton analysis dedicated to MSSM electroweakino searches at $\sqrt{s}=13 \mathrm{TeV}$ of Ref. [19]. This experimental study includes various signal regions defined according to the final-state lepton (electron and muon) multiplicity $N_{\ell}$ and tau lepton multiplicity $N_{\tau}$, the number of opposite-sign same-flavor pairs of leptons $N_{\mathrm{OSSF}}$, same-flavor pairs of leptons $N_{\mathrm{SF}}$ and opposite-sign pairs of leptons $N_{\mathrm{OS}}$, as well as the value of several kinematic variables like the missing transverse energy $E_{T}^{\prime}$, lepton or dilepton transverse momenta, the transverse mass $M_{T}$ of systems made of a lepton and the missing momentum, the stransverse mass $M_{T 2}$ and the invariant mass of various dilepton systems $M_{\ell \ell}$.

The reason for which we choose to recast the CMS multilepton analysis of Ref. [19] stems from the nature of the final state originating from the decay of a charginoneutralino system induced by a $W_{R}$-boson exchange.
However, the electroweakinos originating from resonance production of a much heavier particle are expected to be more boosted, resulting in harder kinematic distributions of the corresponding MSSM signal. In order to maximize the signal versus background ratio, we therefore narrow down our investigations to the signal regions in which the $E_{T}$, $M_{T}, M_{\ell \ell}$ and $M_{T 2}$ kinematical variables are imposed to be larger than some threshold values, instead of lying within a specific window. The overflow bins that we focus on are not necessarily the most sensitive signal regions for MSSM electroweakino searches, but prove to be so in our case. Owing to the Majorana nature of the neutralinos, signal events featuring same-sign dileptons are expected to be copiously produced. However, it turns out that the sensitivity of their corresponding parameter regions is smaller than the one of regions requiring three or four leptons. The properties of the signal regions that are most suitable for probing the different types of spectra considered in this work are summarized in Table V. Whilst it is clear that combining the strengths of several regions would increase the overall sensitivity, this task requires to obtain nonpublic information on uncertainty correlations, so that we prefer to be conservative and focus on one region at a time.

More in details, all the signal regions under category-A require the presence of three light-flavored charged leptons $(\ell \equiv e, \mu)$, with transverse momenta greater than 25 (20) $\mathrm{GeV}$ and 15 (10) $\mathrm{GeV}$ for the leading and subleading leptons respectively, in the electron (muon) case. The pseudorapidity of each electron (muon) is moreover imposed to satisfy $\left|\eta_{\ell}\right|<2.5(2.4)$. The three selected lepton candidates must form at least one opposite-sign same-flavor (OSSF) pair of leptons, and feature a trilepton invariant mass satisfying $\left|m_{3 \ell}-m_{Z}\right|>15 \mathrm{GeV}$. In addition, for the $\mathbf{A 4 4}$ signal region the invariant mass of the dilepton system constructed from the OSSF lepton pair that is as compatible as possible with a $Z$ boson decay is enforced to be larger than $105 \mathrm{GeV}$, and the transverse mass of the system made of the missing momentum and the third lepton is constrained to be larger than $160 \mathrm{GeV}$. Finally, one demands that the missing energy $E_{T}>200 \mathrm{GeV}$.

Signal regions under category-C are dedicated to finalstates that feature two light-flavored charged leptons which fulfill similar requirements as for the ones in category-A, and one hadronic tau with a transverse momentum 
TABLE VI. Number of signal events populating the various signal regions of interest, normalized to an integrated luminosity of $35.9 \mathrm{fb}^{-1}$ at $13 \mathrm{TeV}$ LHC collisions. We show the $W_{R}$ boson production rate (first line) and the background, along with the number of observed events, as reported by the CMS collaboration in Ref. [19]. The numbers in parentheses following the event counts for the most sensitive signal region (D16) indicate the CLs values computed with MADANALYSIS 5 [70,71].

\begin{tabular}{|c|c|c|c|c|c|c|c|}
\hline & BP1 & BP2 & BP3 & BP4 & BP5 & SM & Obs. \\
\hline$\sigma\left(p p \rightarrow W_{R}\right)(\mathrm{fb})$ & 38.12 & 38.12 & 51.54 & 25.58 & 51.54 & - & - \\
\hline A44 & 0.75 & 0.90 & 0.93 & 2.07 & 0.42 & $2.5 \pm 0.8$ & 0 \\
\hline C18 & 0.78 & 0.28 & 1.30 & 0.27 & 0.24 & $1.9 \pm 0.7$ & 1 \\
\hline D16 & $0.85(0.57)$ & $0.94(0.61)$ & $0.77(0.54)$ & $0.43(0.48)$ & $0.43(0.46)$ & $0.06 \pm 0.05$ & 0 \\
\hline G05 & 0.02 & 0.11 & 0.09 & 1.38 & 0.09 & $0.97 \pm 0.32$ & 0 \\
\hline H04 & 0.03 & 0.66 & 0.24 & 1.81 & 0.20 & $1.9 \pm 0.6$ & 1 \\
\hline
\end{tabular}

$p_{T}>20 \mathrm{GeV}$ and a pseudorapidity satisfying $\left|\eta_{\tau}\right|<2.3$. For signal region C18, the two light-flavored leptons are enforced to form an OSSF lepton pair with an invariant mass $M_{\ell \ell}$ larger than $105 \mathrm{GeV}$. The missing transverse energy and the event stransverse mass $M_{T 2}$ are finally imposed to be respectively larger than 200 and $100 \mathrm{GeV}$.

Signal regions under category-D also focus on a topology featuring two light-flavored charged leptons and one hadronic tau. In addition to the previous requirements on the leptons, the $p_{T}$ threshold on the leading light-flavored lepton is increased to $25 \mathrm{GeV}$ when it is a muon and when the subleading light-flavored lepton is an electron. These two leptons are moreover required to be of opposite signs and of different flavors. Event selection for D16 require at least $200 \mathrm{GeV}$ of missing transverse energy and a stransverse mass larger than $100 \mathrm{GeV}$, the variable being constructed from the $e \mu$ pair.

Signal regions under category-G focus on final states containing at least four light-flavored charged leptons (with transverse momentum and pseudorapidity requirements as above), and no hadronic tau. One requires these leptons to form at least two OSSF lepton pairs and for G05, the events should satisfy $E_{T}>200 \mathrm{GeV}$. Signal regions under category-H are similar, except that they require at most one OSSF lepton pair and for $\mathbf{H 0 4}, E_{T}>200 \mathrm{GeV}$.

In the following, we estimate the LHC sensitivity to our LRSUSY setups by investigating how the five abovementioned signal regions are populated by the $W_{R}$ boson mediated electroweakino signal. To this aim, we have used a SARAH 4 implementation of the model $[17,53]$ to be able both to generate the particle spectrum with SPHENO 3 [54], as already above-mentioned, and to export the model information under the form of an LRSUSY UFO library [52] to be used with MADDM 2.0 [51] (for DM calculations) and MG5_AMC@NLO v2.5.5 [68] (for hard scattering LHC event generation). Our computations rely on the leading order set of NNPDF 2.3 parton distribution functions [69]. The simulation of the LHC QCD environment has been achieved with PYTHIA 8 [67] and we have made use of MADANALYSIS 5 [70,71] to automatically handle the impact of the CMS detector with DeLPHES 3 [72] and event reconstruction with FASTJET 3.3.0 [73]. We have reinterpreted the results of the CMS analysis of Ref. [19] by relying on the MADANALYSIS 5 reimplementation of this analysis [74], available from the MADANALYSIS Public Analysis Database [75,76].

\section{B. Results}

Using the methods presented at the end of Sec. VI A, we present our results, for $35.9 \mathrm{fb}^{-1}$ of LHC collisions at a center-of-mass energy of $13 \mathrm{TeV}$, in Tables VI and VII. Table VI shows our predictions for the number of signal events expected to populate the five considered signal regions, for an integrated luminosity of $35.9 \mathrm{fb}^{-1}$ at $13 \mathrm{TeV}$ LHC collisions. Those results can be compared with the SM expectation as extracted from Ref. [19] (last column). Table VII shows estimates for the signal significance defined as

TABLE VII. Statistical significance $\mathcal{S}=S / \sqrt{S+B+\sigma_{B}^{2}}$ when 300 and $3000 \mathrm{fb}^{-1}$ of $13 \mathrm{TeV}$ LHC collisions are considered, for the five benchmarks under investigation. The level of background uncertainty $\sigma_{B} / B$ is assumed equal to the $35.9 \mathrm{fb}^{-1} \mathrm{case}$ of Ref. [19].

\begin{tabular}{lcccrr}
\hline \hline & BP1 & BP2 & BP3 & BP4 & BP5 \\
\hline $\mathcal{L}$ & $300(3000) \mathrm{fb}^{-1}$ & $300(3000) \mathrm{fb}^{-1}$ & $300(3000) \mathrm{fb}^{-1}$ & $300(3000) \mathrm{fb}^{-1}$ & $300(3000) \mathrm{fb}^{-1}$ \\
A44 & $0.74(0.91)$ & $0.88(1.09)$ & $0.91(1.13)$ & $1.90(2.50)$ & $0.42(0.51)$ \\
C18 & $0.87(1.08)$ & $0.32(0.39)$ & $1.39(1.79)$ & $0.31(0.38)$ & $0.28(0.33)$ \\
D16 & $2.55(7.35)$ & $2.69(7.82)$ & $2.41(6.91)$ & $1.74(4.70)$ & $1.74(4.70)$ \\
G05 & $0.04(0.06)$ & $0.23(0.32)$ & $0.19(0.27)$ & $2.22(3.82)$ & $0.19(0.27)$ \\
H04 & $0.04(0.05)$ & $0.81(1.06)$ & $0.31(0.39)$ & $2.02(2.85)$ & $0.26(0.32)$ \\
\hline \hline
\end{tabular}




$$
\mathcal{S}=\frac{S}{\sqrt{S+B+\sigma_{B}^{2}}}
$$

where $S$ and $B$ stands for the number of signal and background events populating a given signal region, and $\sigma_{B}$ stands for the background uncertainty. Our results are provided for two different luminosity configurations of the LHC, namely 300 and $3000 \mathrm{fb}^{-1}$, and for the five different benchmark points and the five considered signal regions. We have estimated the background contributions by rescaling the expectation of Ref. [19], and we have assumed the same uncertainty as for $35.9 \mathrm{fb}^{-1}$.

From the branching ratio information enlisted in Tables III and IV, it is clear that multilepton production from $W_{R}$ boson decays is important. Trilepton final states (probed by the A44, C18 and D16 regions) can, in principle, originate from both $W_{R} \rightarrow \tilde{\chi}_{i}^{0} \tilde{\chi}_{j}^{ \pm}$and $W_{R} \rightarrow$ $N \ell$ decays. However, in our present scenario, the left-right mixing being extremely small, all the right-handed neutrinos entirely decay via off-shell $W_{R}$ into the three body decay mode $\ell q q^{\prime}$. Thus three or more leptons in the final state can only arise substantially from $W_{R}$ decay into the neutralino-chargino pairs and the subsequent cascades. In the context of the BP1 scenario, all three trilepton regions are roughly similarly populated, with a slight preference for the D16 region. Leptons mostly originate from the decays of the weak bosons produced in the electroweakino cascade decays, although they can directly stem, in some rarer cases, from a $\tilde{\chi}_{2}^{ \pm}$decay into a sneutrino LSP along with an extra (often tau) leptons. Trilepton production in the BP2 context is slightly enhanced compared with the BP1 scenario, thanks to the presence of a light selectron in the spectrum allowing Higgsinos to produce more electrons in their decays. Tau production is additionally ensured via chargino decays, so that the D16 and A44 regions turn out to be better to probe the signal. The $\mathbf{B P 3}$ scenario features a more compressed spectrum and understandably, one requires a lighter $W_{R}$ in order to obtain comparable event rates. $\tau$-enriched final states are more prominent in this case because of the large decay branching ratios of both $\tilde{\chi}_{1}^{ \pm}$and $\tilde{\chi}_{2}^{ \pm}$into $\tau$ modes. In the context of the fourth benchmark BP4, light winos guarantee a substantial trilepton signal, that is easier to detect when the presence of taus is not required. In contrast, the spectrum of the BP5 scenario does not allow for copious multilepton production, rendering this LRSUSY configuration difficult to probe with electroweakino searches.

As shown by the number of events populating the G05 and $\mathrm{HO4}$ regions, four-lepton final states are generally produced substantially. In most cases, four-lepton channels, even if associated with a smaller background, cannot compete with the trileptonic modes. Our scenario BP4 consists however in an exception. Here, charginos mostly decay into the LSP along with electrons, as the LSP sneutrino is of the first generation. Fewer tau leptons thus appear in the cascade, and branchings into four lightflavored leptons are larger.

Moving on with prospects for the high-luminosity LHC, we observe in Table VII that the D16 region proves to be the best handle on all LRSUSY configurations, mainly because the associated background is small. It indeed yields almost a $5 \sigma$ significance to all channels. The D16 search region is the most significant, especially when the LSP consists in a sneutrino of third generation, as chargino decays into taus are more common. Not surprisingly, the BP1 and BP2 scenarios offer best discovery prospects at high luminosity, given the large mass gaps between the

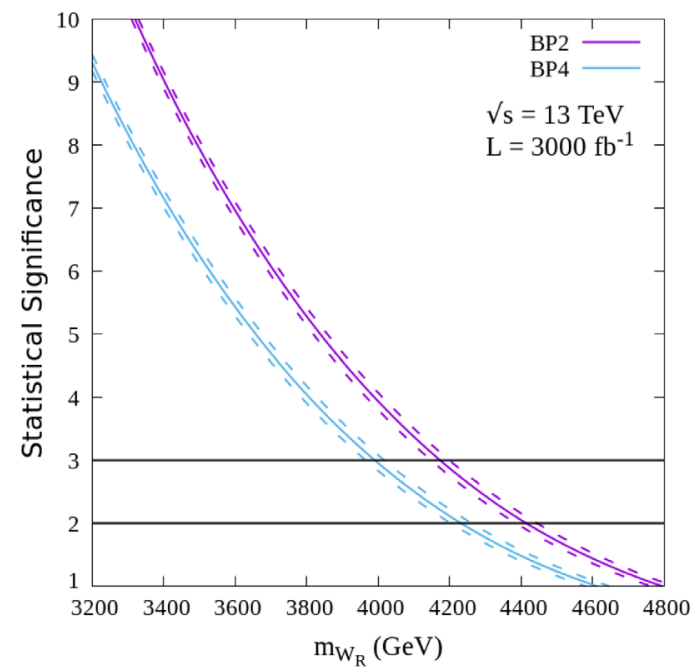

FIG. 4. Statistical significance of the best signal region (D16) for an integrated luminosity of $3000 \mathrm{fb}^{-1}$ and for the five chosen benchmark points. The uncertainties on the background are assumed similar to the current ones (at $35.9 \mathrm{fb}^{-1}$ ) and we study the dependence of the results on the mass of the $W_{R}$ boson. 
neutralino and chargino states and the sneutrino LSP, which results to hard lepton and tau production from the electroweakino cascades. At lower luminosity for which the D16 signal is statistically limited, other signal regions can be more effective, like for example, the four-lepton $\mathbf{G 0 5}$ and H04 modes that are more sensitive to the BP4 scenario featuring large mass splittings that could yield harder leptons than the D16 region.

In Figure 4, we study deviations of our five benchmarks where the mass of the $W_{R}$ boson is varied in the $[3,5] \mathrm{TeV}$ range, with all other model parameters kept unaltered. For our predictions, we solely consider the most sensitive search region, namely the D16 one, and focus on $3000 \mathrm{fb}^{-1}$ of LHC collisions at a center-of-mass energy of $13 \mathrm{TeV}$. The impact of the $W_{R}$ mass on the selection efficiency is of at most $10 \%$, so that we can approximate it to be constant upon the chosen $W_{R}$ boson mass range. Once again, we adopt background relative uncertainties to be equal to those obtained at $35.9 \mathrm{fb}^{-1}$, which yields a conservative estimate on the $m_{W_{R}}$ reach since the ratio $\sigma_{B} / B \simeq 0.83$ is quite large for what concerns the D16 region. We indicate, in the figure, the effect of a $10 \%$ variation on the background uncertainty by dotted lines. Once again, the BP1 and BP2 scenarios offer the best prospects, as $W_{R}$ bosons as heavy as about $4.4-4.5 \mathrm{TeV}$ could be excluded. The sensitivity to the BP3 and BP4 scenarios is also quite encouraging, with an exclusion reach of about 4.2 TeV, and the Higgsino-like LSP BP5 case is again the most complicated to probe.

One can, in principle, get an estimate of the $W_{R}$ boson mass from the decay products. Whilst the final state arises from the production and decay of a resonance, one can construct a transverse variable involving the momenta of all the final state leptons and the missing momentum. Such an observable exhibits a kinematic threshold that could be used to get information on the $W_{R}$ boson mass. The low expected signal statistics could, however, challenge this task, but combining varied search regions could potentially provide extra handles on the new boson.

\section{SUMMARY AND CONCLUSION}

We have considered minimal left-right supersymmetric new physics scenarios and studied dark-matter-motivated configurations. In order to obtain viable DM candidates with respect to the relic density and DM direct detection bounds, we have demonstrated that scenarios quite compressed are in order to guarantee a sufficient level of coannihilations. In particular, scenarios featuring a light sneutrinos LSP can hardly escape being ruled out when the mass splitting between the LSP and the NLSP is large, although they are perfectly viable when the spectrum is compressed. Heavier sneutrinos and bidoublet Higgsino
LSPs are also good options, even if co-annihilation channels are here a requirement (and automatic in the Higgsino case) to ensure the agreement with cosmological data.

We have chosen five benchmark scenarios to showcase some of the features of these co-annihilating LRSUSY DM scenarios and investigated how they could be probed at colliders through multileptonic signals emerging from the production of a $W_{R}$ boson decaying into electroweakinos. Whilst we have mostly focused on a sneutrino LSP coannihilating with wino and Higgsino setup, we have also studied one example of Higgsino LSP for comparison. For each scenario, we have investigated the status with respect to indirect dark matter detection, focusing on the impact of the recent results of the Fermi-LAT collaboration. We have found that the typical DM annihilation cross sections at the present epoch lie two orders of magnitude below the current bounds, so that all scenarios are safe.

We have then moved on with a study of the corresponding multilepton plus missing energy collider signals. The results are very promising, as cosmologically-favorable configuration leads to the production of hard leptons and taus, in association with missing energy, that could be observed through standard electroweakino searches. We have demonstrated that by using one single of the numerous signal regions targeting electroweakinos, the high-luminosity phase of the LHC will allow to collect enough data to (almost) observe any of the considered LRSUSY configurations and thus discover left-right supersymmetry (which requires both the observation of a charged $W_{R}$ boson and a missing energy signal).

\section{ACKNOWLEDGMENTS}

M. F. acknowledges NSERC for partial financial support under Grant No. SAP105354 and thanks the Helsinki Institute of Physics for their hospitality. H. W. acknowledges the financial support from Magnus Ehrnrooth foundation. B. F. has been supported in part by French state funds managed by the Agence Nationale de la Recherche (ANR), in the context of the LABEX ILP (ANR-11-IDEX-0004-02, ANR10-LABX-63). S. M., K. H. and H. W. acknowledge H2020MSCA-RICE-2014 Grant No. 645722 (NonMinimal Higgs). A. C. acknowledges support from DST, India, under Grant No. IFA 15 PH-130. The work of S. K. R. was partially supported by funding available from the Department of Atomic Energy, Government of India, for the Regional Centre for Accelerator-based Particle Physics (RECAPP), Harish-Chandra Research Institute. We thank Tesla Jeltema for her comments on the estimation of Inverse Compton contribution, in comparison to the prompt gammaray flux originating from Dark Matter annihilation in the context of dSPhs. 
[1] Y. Fukuda et al. (Super-Kamiokande Collaboration), Phys. Rev. Lett. 81, 1562 (1998).

[2] A. Aguilar-Arevalo et al. (LSND Collaboration), Phys. Rev. D 64, 112007 (2001).

[3] M. H. Ahn et al. (K2K Collaboration), Phys. Rev. Lett. 90, 041801 (2003).

[4] K. Abe et al. (T2K Collaboration), Phys. Rev. Lett. 107, 041801 (2011).

[5] F. P. An et al. (Daya Bay Collaboration), Phys. Rev. Lett. 112, 061801 (2014).

[6] F. Zwicky, Helv. Phys. Acta 6, 110 (1933) [Gen. Relativ. Gravit. 41, 207 (2009)].

[7] V. C. Rubin and W. K. Ford, Jr., Astrophys. J. 159, 379 (1970).

[8] P. A. R. Ade et al. (Planck Collaboration), Astron. Astrophys. 594, A13 (2016).

[9] D. Clowe, M. Bradac, A. H. Gonzalez, M. Markevitch, S. W. Randall, C. Jones, and D. Zaritsky, Astrophys. J. 648, L109 (2006).

[10] J. C. Pati and A. Salam, Phys. Rev. D 10, 275 (1974); 11, 703(E) (1975).

[11] R. N. Mohapatra and J. C. Pati, Phys. Rev. D 11, 566 (1975).

[12] R. N. Mohapatra and A. Rasin, Phys. Rev. Lett. 76, 3490 (1996).

[13] R. Kuchimanchi, Phys. Rev. Lett. 76, 3486 (1996).

[14] R. N. Mohapatra and A. Rasin, Phys. Rev. D 54, 5835 (1996).

[15] R. Kuchimanchi and R. N. Mohapatra, Phys. Rev. D 48, 4352 (1993).

[16] K. S. Babu and R. N. Mohapatra, Phys. Lett. B 668, 404 (2008).

[17] L. Basso, B. Fuks, M. E. Krauss, and W. Porod, J. High Energy Phys. 07 (2015) 147.

[18] M. Frank, B. Fuks, K. Huitu, S. K. Rai, and H. Waltari, J. High Energy Phys. 05 (2017) 015.

[19] A. M. Sirunyan et al. (CMS Collaboration), J. High Energy Phys. 03 (2018) 166.

[20] A. Alloul, M. Frank, B. Fuks, and M. Rausch de Traubenberg, J. High Energy Phys. 10 (2013) 033.

[21] C. S. Aulakh, K. Benakli, and G. Senjanovic, Phys. Rev. Lett. 79, 2188 (1997).

[22] S. Bhattacharya, E. Ma, and D. Wegman, Eur. Phys. J. C 74, 2902 (2014).

[23] E. Aprile et al. (XENON Collaboration), Phys. Rev. Lett. 121, 111302 (2018).

[24] X. Cui et al. (PandaX-II Collaboration), Phys. Rev. Lett. 119, 181302 (2017).

[25] M. Frank and B. Korutlu, Phys. Rev. D 83, 073007 (2011).

[26] K. S. Babu and A. Patra, Phys. Rev. D 93, 055030 (2016).

[27] M. Aaboud et al. (ATLAS Collaboration), Eur. Phys. J. C 78, 199 (2018).

[28] CMS Collaboration, Report No. CMS-PAS-HIG-16-036, 2017, http://cds.cern.ch/record/2242956.

[29] G. Aad et al. (ATLAS Collaboration), Phys. Lett. B 716, 1 (2012).

[30] S. Chatrchyan et al. (CMS Collaboration), Phys. Lett. B 716, 30 (2012).

[31] K. S. Babu, X.-G. He, and E. Ma, Phys. Rev. D 36, 878 (1987).
[32] K. Huitu, P. N. Pandita, and K. Puolamaki, Phys. Lett. B 423, 97 (1998).

[33] K. S. Babu and C. F. Kolda, Phys. Rev. Lett. 84, 228 (2000).

[34] P. Achard et al. (L3 Collaboration), Phys. Lett. B 517, 75 (2001).

[35] M. Aaboud et al. (ATLAS Collaboration), Phys. Rev. D 96, 052004 (2017).

[36] A. M. Sirunyan et al. (CMS Collaboration), J. High Energy Phys. 08 (2018) 130.

[37] M. Aaboud et al. (ATLAS Collaboration), J. High Energy Phys. 10 (2017) 182.

[38] A. M. Sirunyan et al. (CMS Collaboration), J. High Energy Phys. 06 (2018) 120.

[39] W.-Y. Keung and G. Senjanovic, Phys. Rev. Lett. 50, 1427 (1983).

[40] A. M. Sirunyan et al. (CMS Collaboration), J. High Energy Phys. 05 (2018) 148.

[41] M. Aaboud et al. (ATLAS Collaboration), J. High Energy Phys. 01 (2019) 016.

[42] A. M. Sirunyan et al. (CMS Collaboration), J. High Energy Phys. 07 (2017) 121.

[43] P. S. Bhupal Dev, S. Mondal, B. Mukhopadhyaya, and S. Roy, J. High Energy Phys. 09 (2012) 110.

[44] V. De Romeri and M. Hirsch, J. High Energy Phys. 12 (2012) 106.

[45] S. Banerjee, P. S. B. Dev, S. Mondal, B. Mukhopadhyaya, and S. Roy, J. High Energy Phys. 10 (2013) 221.

[46] J. Chang, K. Cheung, H. Ishida, C.-T. Lu, M. Spinrath, and Y.-L. S. Tsai, J. High Energy Phys. 09 (2018) 071.

[47] N. Arkani-Hamed, L. J. Hall, H. Murayama, D. Tucker-Smith, and N. Weiner, Phys. Rev. D 64, 115011 (2001).

[48] B. Dumont, G. Belanger, S. Fichet, S. Kraml, and T. Schwetz, J. Cosmol. Astropart. Phys. 09 (2012) 013.

[49] A. Chatterjee, D. Das, B. Mukhopadhyaya, and S. K. Rai, J. Cosmol. Astropart. Phys. 07 (2014) 023.

[50] C. Arina, M. E. C. Catalan, S. Kraml, S. Kulkarni, and U. Laa, J. High Energy Phys. 05 (2015) 142.

[51] M. Backović, A. Martini, O. Mattelaer, K. Kong, and G. Mohlabeng, Phys. Dark Universe 9-10, 37 (2015).

[52] C. Degrande, C. Duhr, B. Fuks, D. Grellscheid, O. Mattelaer, and T. Reiter, Comput. Phys. Commun. 183, 1201 (2012).

[53] F. Staub, Comput. Phys. Commun. 185, 1773 (2014).

[54] W. Porod and F. Staub, Comput. Phys. Commun. 183, 2458 (2012).

[55] J. Edsjo and P. Gondolo, Phys. Rev. D 56, 1879 (1997).

[56] S. Profumo and A. Provenza, J. Cosmol. Astropart. Phys. 12 (2006) 019.

[57] M. Aguilar et al. (AMS Collaboration), Phys. Rev. Lett. 117, 091103 (2016).

[58] A. Chatterjee, M. Frank, B. Fuks, K. Huitu, S. Mondal, S. K. Rai, and H. Waltari, http://inspirehep.net/record/1712932/.

[59] T. Bringmann and C. Weniger, Phys. Dark Universe 1, 194 (2012).

[60] J. Lavalle and P. Salati, C.R. Phys. 13, 740 (2012).

[61] S. Profumo and P. Ullio, arXiv:1001.4086.

[62] M. Ackermann et al. (Fermi-LAT Collaboration), Phys. Rev. Lett. 115, 231301 (2015). 
[63] M. Ackermann et al. (Fermi-LAT Collaboration), Astrophys. J. 840, 43 (2017).

[64] M. D. Campos, F. S. Queiroz, C. E. Yaguna, and C. Weniger, J. Cosmol. Astropart. Phys. 07 (2017) 016.

[65] T. Bringmann et al. (The GAMBIT Dark Matter Workgroup), Eur. Phys. J. C 77, 831 (2017).

[66] A. McDaniel, T. Jeltema, S. Profumo, and E. Storm, J. Cosmol. Astropart. Phys. 09 (2017) 027.

[67] T. Sjöstrand, S. Ask, J. R. Christiansen, R. Corke, N. Desai, P. Ilten, S. Mrenna, S. Prestel, C. O. Rasmussen, and P.Z. Skands, Comput. Phys. Commun. 191, 159 (2015).

[68] J. Alwall, R. Frederix, S. Frixione, V. Hirschi, F. Maltoni, O. Mattelaer, H. S. Shao, T. Stelzer, P. Torrielli, and M. Zaro, J. High Energy Phys. 07 (2014) 079.

[69] R. D. Ball et al. (NNPDF Collaboration), J. High Energy Phys. 04 (2015) 040.
[70] E. Conte, B. Fuks, and G. Serret, Comput. Phys. Commun. 184, 222 (2013).

[71] E. Conte, B. Dumont, B. Fuks, and C. Wymant, Eur. Phys. J. C 74, 3103 (2014).

[72] J. de Favereau, C. Delaere, P. Demin, A. Giammanco, V. Lemaitre, A. Mertens, and M. Selvaggi (DELPHES 3 Collaboration), J. High Energy Phys. 02 (2014) 057.

[73] M. Cacciari, G. P. Salam, and G. Soyez, Eur. Phys. J. C 72, 1896 (2012).

[74] B. Fuks and S. Mondal, https://inspirehep.net/record/ $1676304 /$.

[75] B. Dumont, B. Fuks, S. Kraml, S. Bein, G. Chalons, E. Conte, S. Kulkarni, D. Sengupta, and C. Wymant, Eur. Phys. J. C 75, 56 (2015).

[76] E. Conte and B. Fuks, Int. J. Mod. Phys. A 33, 1830027 (2018). 